\title{
Temperature and heat flux fast estimation during rolling process
}

\author{
Daniel Weisz-Patrault ${ }^{\mathrm{a}}$, Alain Ehrlacher ${ }^{\mathrm{a}}$, Nicolas Legrand ${ }^{\mathrm{b}}$ \\ ${ }^{a}$ Ecole Ponts ParisTech, UR Navier, 6 \& 8 Ave Blaise Pascal, 77455 Marne La Vallee, France \\ ${ }^{b}$ ArcelorMittal Global Research \& Development, Maizière Process Voie Romaine, BP 30320 F-57283 Maizières-lès-Metz Cedex
}

\begin{abstract}
Monitoring and controlling flatness during the rolling process becomes critical for ensuring the product quality. Flatness defects are due to highly three-dimensional phenomena. Indeed, strips with different widths are rolled during the same campaign and cooling systems are heterogeneous along the axial direction to modify the thermal expansion of the roll. Therefore this paper presents a fully three-dimensional inverse analytical method to determine the temperature field and heat fluxes (especially at the surface of the roll) by interpreting measurements of temperature done with several thermocouples fully embedded in the roll body and aligned along the axial direction. Since the method is dedicated to on-line interpretation and designed as a tool for adapting the rolling parameters during the rolling process, iterative methods are not studied to avoid long computation times, which justifies the development of an analytical solution of the problem. The computation time displayed by Scilab 5.3 with a quadcore $2.8 \mathrm{GHz}$ is around 0.5 second by cycle for accurate computation and 0.07 second by cycle for rough computation. This paper improves a previous work $(2 \mathrm{D}$ and relying on four assumptions designed for the prediction of wear). In the present contribution the 3D unsteady heat equation of the rotating roll is solved analytically with only one assumption in order to deal with the restriction of the measurement system (i.e., measurement according to successive times). Therefore not only radial and tangential heat fluxes are taken into account but also axial heat flux. The solution is validated by comparing the outputs of the method and some prescribed analytical temperature fields. Good agreement is obtained. Noise sensitivity is estimated by adding artificial random numbers to the inputs, and good accuracy is observed. Moreover sensitivity to sensor depth is estimated and demonstrated to be not compromising.
\end{abstract}

Keywords:

Inverse conduction, Inverse method, Meshless, Heat flux, Temperature, Analytic, Flatness, Rolling process

\begin{tabular}{|ll|}
\hline$R_{S}$ & Outer radius (radius of the surface of the roll) \\
$R_{m}$ & Inner radius (radius of the measurements) \\
$e$ & Error of the sensor depth \\
$\omega$ & Rotation speed \\
$r$ & Radial position \\
$\theta$ & Angular position \\
$z$ & Axial position \\
$t$ & Time \\
$k$ & Index of the current cycle \\
$t_{k}$ & Time at the beginning of the $k$ th cycle $\left(=t_{k-1}+2 \pi / \omega\right)$ \\
$t_{\theta}^{k}$ & Time related to the angular position $\left(=t_{k}+\theta / \omega\right)$ \\
$f$ & Frequency of acquisition of the measurements \\
$\lambda$ & Thermal conductivity of the roll \\
$D$ & Thermal diffusivity of the roll \\
$\epsilon$ & Percentage of error \\
$T$ & Temperature field (solution) \\
$\boldsymbol{H}$ & Heat flux field (solution) \\
$T_{1}$ & First part of the temperature field (solution) \\
$T_{2}$ & Second part of the temperature field (solution)
\end{tabular}




\begin{tabular}{|c|c|}
\hline$T^{m}$ & Measured temperatures (inputs) \\
\hline$A_{n, p}^{k}$ & $n, p$ th cosine coefficient of Fourier of $T^{m}$ ( $k$ th cycle) \\
\hline$B_{n, p}^{k}$ & $n, p$ th sine coefficient of Fourier of $T^{m}(k$ th cycle $)$ \\
\hline$T^{s}$ & Temperature at the surface of the roll (outputs) \\
\hline $\boldsymbol{H}^{s}$ & Heat flux at the surface of the roll (outputs) \\
\hline$T^{p}$ & Prescribed temperature field (validation of the method) \\
\hline$T_{a}$ & Ambient temperature \\
\hline$T^{*}$ & Surrounding temperature (validation of the method) \\
\hline$a_{n, p}^{*}$ & $n, p$ th cosine coefficient of Fourier of $T^{*}$ \\
\hline$b_{n, p}^{*}$ & $n, p$ th sine coefficient of Fourier of $T^{*}$ \\
\hline HTC & Heat Transfer Coefficient (validation of the method) \\
\hline$N_{1}$ & Order of truncation (integer) \\
\hline$N_{2}$ & Order of truncation (integer) \\
\hline$P_{1}$ & Order of truncation (integer) \\
\hline$P_{2}$ & Order of truncation (integer) \\
\hline$Q_{1}$ & Order of truncation (integer) \\
\hline$Q_{2}$ & Order of truncation (integer) \\
\hline$S$ & Number of thermocouples (integer) \\
\hline$N_{\theta}$ & Number of reconstruction points along circumferential direction \\
\hline$N_{z}$ & Number of reconstruction points along axial direction \\
\hline$N_{\theta}^{i}$ & Number of interpolation points along circumferential direction \\
\hline$N_{z}^{i}$ & Number of interpolation point along axial direction \\
\hline$\zeta_{n, p}$ & Coefficients (complex) \\
\hline$\chi_{n, p}$ & Coefficients (complex) \\
\hline$J_{n}$ & Bessel function of the first kind of the order $n$ \\
\hline$\alpha_{n, p, q}^{(k)}$ & Coefficients (complex) \\
\hline$\beta_{n, p, q}^{(k)}$ & Coefficients (complex) \\
\hline$a_{n, p}$ & Coefficients (complex) \\
\hline$b_{n, p}$ & Coefficients (complex) \\
\hline$c_{n, p, q}$ & Coefficients (complex) \\
\hline$d_{n, p, q}$ & Coefficients (complex) \\
\hline$h_{n}$ & Auxiliary function \\
\hline$x_{n, q}$ & Successive positive zeros of $J_{n}$ \\
\hline$y_{n, q}$ & Successive zero of $h_{n}$ \\
\hline$\gamma$ & Coefficient (complex) \\
\hline$\tau$ & Relaxation time (complex) \\
\hline$\tau_{n, p, q}$ & Relaxation time (complex) \\
\hline$v_{n, p, q}$ & Relaxation time (complex) \\
\hline$\tau_{n, p, q}^{*}$ & Relaxation time (complex) \\
\hline$v_{n, p, q}^{*}$ & Relaxation time (complex) \\
\hline$\Theta$ & Angle \\
\hline $\boldsymbol{u}$ & Displacement field \\
\hline$\left(\lambda_{0}, \mu_{0}\right)$ & Lame's coefficients of the roll \\
\hline
\end{tabular}

Table 1: Nomenclature

\section{Introduction}

\subsection{Context}

In steel rolling processes, two rolls are used as tools to reduce the thickness of a workpiece. Flatness control improvement is essential for productivity, automation and quality, since the requirements for strip crown and flatness are more and more severe. Flatness defects origin is the difference between the incoming strip profile and the work roll deformed profile. The cooling system as well as crown control devices for shape correction are voluntary heterogeneous along the axial direction in order to compensate the heterogeneous temperature fields. Moreover a rolling campaign involves often many different strip widths. Therefore the mechanisms involved in flatness problems are highly three-dimensional. 
Predictive models are very useful for the design of flatness control devices and cooling system. Thus many studies focus on more and more comprehensive approaches of flatness during rolling process. A complete flatness model combines thermo-mechanic models for the strip and thermo-elastic models for the roll. For example Jiang and Tieu [1] developed a predictive 3D finite element method (FEM) which gives the contact stress between the strip and the roll, the deformation of the roll and especially the shape of the roll generatrix and by taking into account the shape of the incoming strip gives the longitudinal stress profile of the outcoming strip. More recently Abdelkhalek et al. [2] proposed a comprehensive FEM which moreover takes into account the buckling of the outcoming strip and the coupling between plastic deformation of the strip in the roll gap and the buckling of the outcoming strip.

All the predictive models of flatness need the computation of the work roll deformation and especially the thermal expansion. Therefore three-dimensional temperature fields should be computed, most of the time by numerical methods. Several authors focus on this latter task. For example Abbaspour and Saboonchi [3] proposed predictive models for the optimisation of cooling system. Thermal crown has been investigated by Zhang et al. [4] and [5] with two dimensional FEM (radial and axial directions) by neglecting circumferential direction. Truly three-dimensional predictive models have also been proposed by Zone-Ching and Chang-Cheng [6] or more recently by $\mathrm{Li}$ et al. [7]. Montmitonnet [8] gave also a comprehensive review of predictive three-dimensional models (by FEM and FDM) of the whole rolling process, by coupling the strip and the roll thermal behaviours with iterative methods.

All these simulations consider very complex boundary conditions. Indeed, the contact between the strip (from around $300 \mathrm{~K}$ for cold rolling conditions to around $1200 \mathrm{~K}$ for hot rolling conditions) and the roll (initially at the room temperature) is responsible for conducting heat, which is often modeled with a heat transfer coefficient. This model parameter is often assumed to be constant in the contact like in the model of Corral et al. [9]. But since pressures are not constant in the contact, this heat transfer coefficient is actually not constant as demonstrated by Legrand et al. [10]. The heat flux entering the roll by conduction from the strip can also be modeled by a heat flux like in the work of Hacquin [11]. Moreover, the cooling and lubrication systems involve a forced convection at the entry and exit of the roll gap and the surrounding air is responsible for a free convection. To a lesser extend, the friction between the strip and the roll, the plastic deformations of the strip and radiations from the strip, are also heat sources for the roll. This complicated thermal problem is summarized in Figure 1.

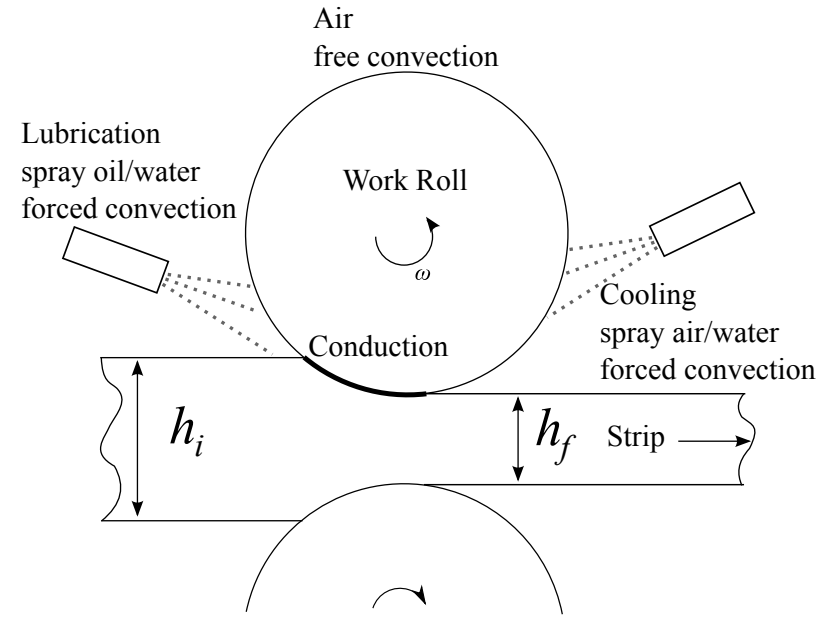

Figure 1: Thermal conditions during rolling process

The idea of this paper is to avoid such a complicated model by replacing this kind of direct and theoretical computations of three-dimensional temperature and heat flux fields inside the roll, by a real-time evaluation based on temperature measurements done with several thermocouples fully embedded inside the roll body. This evaluation is performed by inverse analysis and does not require external conditions around the roll. Temperatures and heat fluxes at the surface are directly inferred from the temperatures measured inside the roll, regardless of contact, cooling and lubrication conditions at the surface.

Most of inverse methods consider boundary conditions at the surface of the roll and compute the temperature at the radius where temperatures are measured. Then a minimization between the computed temperature and 
measured temperature is performed in order to identify the boundary conditions. The present inverse solution does not belong to this class of methods. Indeed, no boundary conditions are considered at the surface, and there is no minimization process. Considering that temperatures are measured at the radius $R_{m}<R_{s}$ (where $m$ means measured and $s$ means surface), a fully analytical solution of the problem is found in a sub-domain (which the cylinder of radius $R_{m}$ ). Then, this analytical solution is extended by continuity toward the surface. In this way, temperature and heat flux fields are deduced everywhere in the roll and especially at the surface without considering any boundary condition at the surface of the roll.

\subsection{Review of inverse methods}

Many authors focus on 1D or 2D inverse methods. (This is due to the fact that most of the time numerical computations are used, and therefore 3D inverse methods involve high stack memory capacity and long CPU times). Among the latest contributions, Volle et al. [12] proposed a 1D analytical inverse method compared with a $2 \mathrm{D}$ numerical inverse method adapted to a hot rotating cylinder cooled by a water jet. Kotrbacek et al. [13] proposed a comprehensive 2D inverse method, where the sensor and temperature dependance of thermal properties are modeled. However computation times are long. More recently Chen and Yang [14] proposed a 2D (radial and circumferential directions) steady approach (with a refined mesh near the roll gap) based on the conjugate gradient algorithm. However the computation times do not allow a real time evaluation, and the time dependence is neglected. Moreover a recent two-dimensional semi-analytical inverse method adapted to rotating cylinders has been proposed by Volle et al. [15] and used by Volle et al. [16] and Gradeck et al. [17]. The method is based on Fourier and Laplace transforms of the unsteady heat equation, then the deconvolution is performed with a numerical algorithm. From unknown boundary conditions assumed to be constant during each time step, the expression of temperature is established at the radius where the measurements are done by means of a sensitivity matrix representing the linear relationship between the unknown heat flux at the surface and the temperature at the radius where the temperature are measured. However, practically this sensitivity matrix is ill-conditionned, therefore a minimization, between the measured and calculated temperatures, is performed with least square methods and regularized with classical techniques. This work has been tested on cooling conditions not very singular compared with common industrial rolling conditions. Furthermore 24 thermocouples are considered along the circumferential direction.

Weisz-Patrault et al. [18] published very recently a fully analytical 2D (radial and circumferential directions) inverse method considering only one thermocouple embedded inside the roll, and deals with rolling conditions. The method is very fast $(0.05 \mathrm{sec}$ by cycle) and relies on the $2 \mathrm{D}$ unsteady heat equation. Weisz-Patrault et al. [19] proposed also an experimental study to show how the thermocouple can be inserted inside the roll and how it can be calibrated. The wires are evacuated by the center of the cylinder and signals are transmitted by inductive system. The paper focus also on the effect of the reduction of the strip. Legrand et al. [20] proposed an other experimental study (based on the inverse method [18]), which focuses on the influence of scale thickness and contact resistance. Moreover a 2D analytical inverse method published recently by Weisz-Patrault et al. [21] for the evaluation of contact stress during the rolling process can be combined with the thermal inverse method [18] in order to obtain the analytical 2D thermal stresses, which has been published by Weisz-Patrault et al. [22]. A fast on-line experimental evaluation of thermal fatigue is therefore possible.

\section{Industrial interest of 3D inverse method}

The general motivation of this paper is to improve the knowledge of real three-dimensional temperature and heat flux fields in the work roll, occurring during rolling process. Indeed, if theoretical works, which have been cited in section 1.1, enable a good understanding of physical phenomena involved in the rolling process, and are therefore the dedicated tools for the design of control devices, they cannot replace experimental evaluation of real temperature and heat flux fields. Moreover, the on-line monitoring of temperature fields could improve significantly the use of cooling systems and flatness control devices, by adapting them in real time with a closeloop control. Therefore this paper aims at developing the basis of an on-line industrial tool for evaluating industrial temperature fields in real time during the rolling process by interpreting some temperature measurements done with several thermocouples aligned along the axial direction and fully embedded inside the roll body (see Fig.2).

As mentioned above temperature and heat flux fields are very heterogenous along the axis of the roll. That is why Keanini [23] proposed a 3D numerical inverse model, by inserting several thermocouples at different locations in the roll. The circumferential direction is not considered in the equations, the tangential dependence is only obtained with the rotation of the roll. Loulou and Artioukhine [24] developed a truly 3D inverse method 
interpreting measurements of several thermocouples, adapted for hollow cylinders and based on the iterative regularization method and residual functional minimization by using the unconstrained conjugate gradient method with the regularizing discrepancy principle. However the CPU times are very long, and the work is done for a non-rotating roll.

In order to determine by inverse analysis the heterogenous fields along the axis of the roll, the authors have basically two choices. The first one consist in applying several times the 2D inverse method previously developed by Weisz-Patrault et al. [18] at different axial locations (corresponding to the axial location of each thermocouple). The second choice is the development of a truly 3D analytical inverse method. This latter solution has been chosen and the interest of a truly 3D inverse method compared with a 2D inverse method applied several times should be highlighted.

A single computation gives, for the present 3D inverse method, the analytical temperature and heat flux fields in the whole roll, whereas the 2D inverse method used several times at the different axial locations (where thermocouples are inserted) gives only the temperature and heat flux fields at these axial locations. The former solution allows to compute analytically the thermal expansion including shear stress, when the latter allows only to compute the thermal expansion for different sections of the roll assumed to be independent from each other (i.e. neglecting shear stress in the roll), which is done for example by Hacquin [11] or Zone-Ching and Chang-Cheng [6].

In the present work the unsteady 3D heat equation is not simplified. All heat fluxes in all directions are taken into account, which is not the case for a 2D inverse method where axial heat fluxes are neglected. This allows the computation of the thermal expansion of the work roll (essential for flatness control) by solving the Navier's equation (1) which involves all the components of the heat flux in the right side term.

$$
\mu_{0} \operatorname{div} \operatorname{grad} \boldsymbol{u}+\left(\lambda_{0}+\mu_{0}\right) \operatorname{grad} \operatorname{div} \boldsymbol{u}=\alpha\left(3 \lambda_{0}+2 \mu_{0}\right) \operatorname{grad} T
$$

The lame's coefficients are denoted by $\mu_{0}$ and $\lambda_{0}$, these elastic coefficients have nothing to do with $\lambda$ which is the thermal conductivity of the roll, and $\boldsymbol{u}$ is the displacement field of the roll and $\alpha$ the thermal dilatation.

Another reason for developing the present 3D inverse method is a significant improvement of the analytical solution of the previous work of Weisz-Patrault et al. [18]. Indeed, the initial condition is verified without any assumption in the present paper when three unnecessary assumptions have been introduced in the previous work [18]. A comparison between the present inverse solution and the 2D inverse solution of Weisz-Patrault et al. [18] is given for different axial positions in section 9. A slightly better accuracy is demonstrated for the present 3D inverse method.

Moreover, using several times the 2D inverse method at different axial locations is not more efficient in terms of computation times (around 1s/cycle for 30 thermocouples) than the present 3D inverse method, which gives much more complete informations (temperature and heat flux fields everywhere in the roll) in $0.5 \mathrm{~s} / \mathrm{cycle}$ (for accurate computation) or $0.07 \mathrm{~s} / \mathrm{cycle}$ (for rough computation).

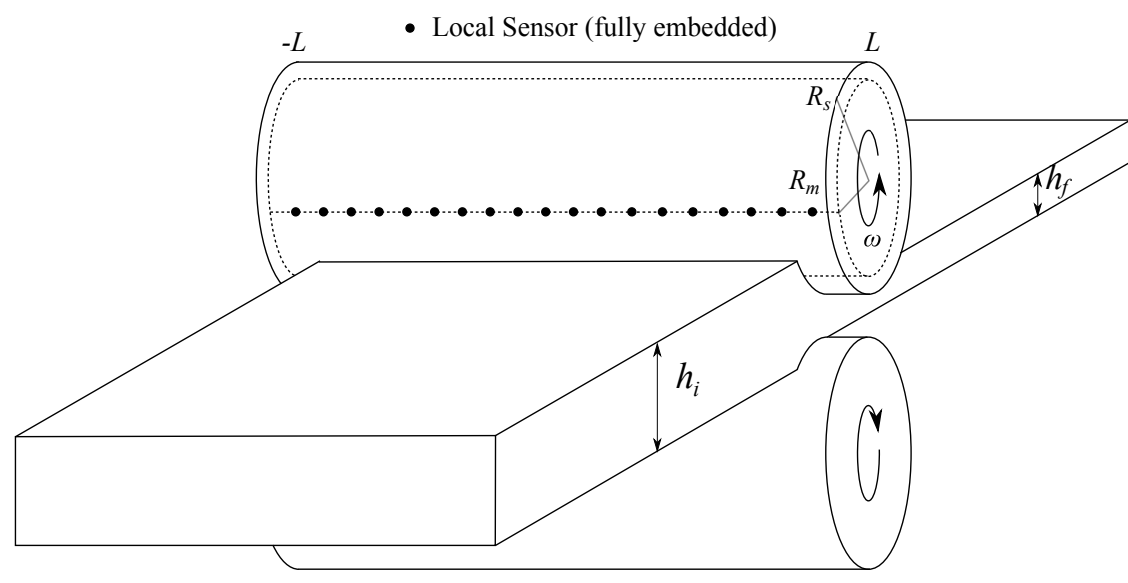

(a) Measurement system

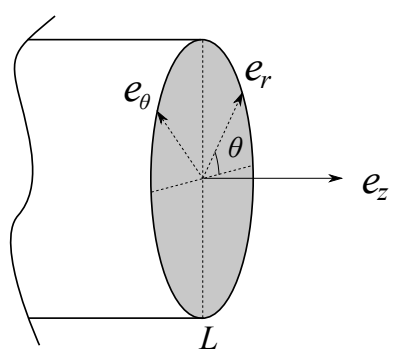

(b) Axes

Figure 2: Rolling process 


\section{Validation of the method}

The inputs consist in several temperature signals delivered by thermocouples aligned along the axial direction (at locations $z_{j}$ where $j \in\{1 ; \ldots ; S\}$ ) and located at the radius $R_{m}$ with $R_{m}<R_{s}$ (where $m$ means measured and $s$ means surface). Temperature signals are obtained for successive times (called $t_{\theta}^{k}=t_{k}+\theta / \omega$ ) according to the rotation speed of the roll and the index $k$ of the cycle. Then cubic splines are used to interpolate the input signal along both the circumferential and axial directions. The outputs are in general the whole analytical temperature field and therefore the radial, circumferential and axial heat fluxes. The temperature and heat fluxes are obtained especially at the surface of the roll.

Considering the temperature in the whole roll, an analytical expansion into series (solution of the unsteady heat equation) is written. The identification of coefficients of the series is done with the conditions available, which are in the present case the temperatures measured inside the roll and the initial condition (room temperature). Therefore the method only consist in finding an analytical solution of the problem in a sub-domain being the cylinder of radius $R_{m}$, then the analytical solution is extended by continuity toward the surface of the roll. There is no iterative matching process and no matrix inversion. Therefore all the classic problems of inverse methods related to ill-posed problems are therefore avoided. (Regularization is done by truncation of series). The frequency of acquisition of the thermocouples is not limited by the method as for Raynaud and Bransier [25].

No experimental data were available. The validation of the method is done as follows. An analytical unsteady temperature field is prescribed in the roll. This field is established in Appendix B and corresponds to a roll heated at the surface in a small angular part corresponding to a contact with a hot steel strip. The resulting temperature field is close from typical temperature fields that occur during rolling processes as observed with the direct predictive model of Li et al. [7] for instance. A numerical simulation could have been used, but for simplicity this simple temperature field has been used instead.

The inputs of the inverse method presented in this paper (replacing measurements) are extracted from this prescribed temperature field. The solution is validated by comparing the outputs of the method and the prescribed analytical temperature at the surface of the roll. Heterogeneous prescribed temperature field along the axial direction is chosen to show the ability of the method to deal with such a difficulty. The relative difference between the outputs and the prescribed temperature is used to quantify the quality of the method. Excellent agreement is obtained. Noise sensitivity is estimated by adding random numbers to the inputs and good accuracy is observed.

\section{Principles of the inverse method}

The evolution of the temperature of a material point (Lagrangian description) of the roll is very fast. The time dependence could be difficult to describe, that is why an Eulerian description is developed in order to have a slow evolution of the temperature field (small variations from one cycle to another). Thus, an Eulerian point ( $r$ radial position, $\theta$ angular position and $z$ axial position) is not material, that is to say it describes the evolution of a succession of material points at a given position in a fixed reference. In the following, all the quantities are calculated in an Eulerian reference.

The temperature measurements are done with respect to time (temperature known at different angular positions at successive times). Each rotation of the roll allows temperature information on the whole cylinder of radius $R_{m}$, but not at all times. Each angular position is known at one particular time according to the frequency of acquisition $f(\mathrm{~Hz})$ and the rotation speed $\omega$.

Therefore, a natural characteristic time of the problem is the duration of each cycle. Thus, cycles are numbered (index $k$ ) and it is convenient to divide the total time interval in subintervals $\left[t_{k}, t_{k+1}\right]$ where $t_{k}$ is the time at the beginning of the $k$ th cycle therefore: $t_{k}=t_{k-1}+2 \pi / \omega$ (considering that the rotation speed $\omega(\mathrm{rad} / \mathrm{s}$ ) is quite constant during each cycle, it should be noted $w_{k}$, but for simplicity it is noted $\omega$ ). Inputs are called $T^{m}(\theta, z, k)$ where $m$ means measured. Notations are listed in Table 1.

The solution of the unsteady heat equation is denoted by $T(r, \theta, z, t, k)(r, \theta$ and $z$ being the radial, angular and axial coordinates, $t$ meaning time and $k$ being the index of the cycle). It should be noted that $t$ and $k$ are related $\left(t \in\left[t_{k}, t_{k+1}\right]\right)$. The thermal diffusivity is denoted by $D\left(\mathrm{~m}^{2} / \mathrm{s}\right)$ which is assumed to be independent on the temperature. The unsteady heat equation governing the temperature field $T(r, \theta, z, t, k)$ during the $k$ th cycle is:

$$
\frac{\partial^{2} T}{\partial r^{2}}+\frac{1}{r} \frac{\partial T}{\partial r}+\frac{1}{r^{2}} \frac{\partial^{2} T}{\partial \theta^{2}}+\frac{\partial^{2} T}{\partial z^{2}}=\frac{1}{D}\left(\frac{\partial T}{\partial t}+\omega \frac{\partial T}{\partial \theta}\right)
$$


With the set of conditions:

$$
\left\{\begin{array}{l}
(r, \theta, z, t, k) \in\left[0, R_{s}\right] \times\left[0,2 \pi\left[\times[-L, L] \times\left[t_{k}, t_{k+1}\right] \times \mathbb{N}^{*}\right.\right. \\
T\left(r, \theta, t_{k}, z, k\right)=\left\{\begin{array}{ll}
T_{a} & k=1 \\
T\left(r, \theta, t_{k}, z, k-1\right) & k \geqslant 2
\end{array} \quad\right. \text { (initial condition) } \\
T\left(R_{m}, \theta, z, t_{\theta}^{k}, k\right)=T^{m}(\theta, z, k) \text { (measured condition) } \\
\frac{\partial T}{\partial z}(r, \theta, \pm L, t, k)=0
\end{array}\right.
$$

For the first cycle, the initial condition is the ambient temperature in the entire roll. For the next cycles the initial condition is the temperature field at the end of the previous cycle. Moreover the roll is much wider than the strip, the edges of the roll are far from the main heat source, therefore it is reasonable to assume that the axial heat flux vanishes at the edges of the roll $(z= \pm L)$, as in the work of Keanini [23]. The latter condition is automatically verified as demonstrated in Section 6, therefore this condition will not appear any more.

It should be noticed that there is no condition at the outer radius $R_{s}$, since temperature and heat fluxes at the surface are unknown and should be evaluated by the inverse method. Usually, a thermal problem needs proper boundary conditions at the surface of the body. However, in this approach, temperatures and heat fluxes at the surface of the roll are determined from measured temperatures at the inner radius $R_{m}$, regardless of external conditions. This is done by splitting the domain into two sub-domains $\mathcal{D}_{d}$ and $\mathcal{D}_{i}(d$ and $i$ meaning direct and inverse):

$$
\left\{\begin{array}{l}
\mathcal{D}_{d}=\left[0, R_{m}\right] \times[0,2 \pi[\times[-L, L] \\
\left.\left.\mathcal{D}_{i}=\right] R_{m}, R_{s}\right] \times[0,2 \pi[\times[-L, L]
\end{array}\right.
$$

The first sub-domain is called the direct part and is the cylinder of radius $R_{m}$. On this sub-domain, the boundary is at radius $r=R_{m}$ and the measured condition $T\left(R_{m}, \theta, z, t_{\theta}^{k}, k\right)=T^{m}(\theta, z, k)$ can be expressed as a proper boundary condition (managing a single assumption discussed in Section 6.2). Thus, it is possible to determine the analytical temperature and heat flux fields in this sub-domain. The second sub-domain is called the inverse part and is the hollow cylinder of inner radius $R_{m}$ and outer radius $R_{s}$. The idea of the method is to extend by continuity the analytical solution determined in the direct part $\mathcal{D}_{d}$ into the second sub-domain $\mathcal{D}_{i}$, including the surface of the roll. This extension by continuity toward the surface enables to not consider any boundary condition at the surface of the roll at the outer radius $R_{s}$. Two new sets of conditions equivalent to 3 are defined:

$$
\begin{aligned}
& \text { (Direct part): }\left\{\begin{array}{l}
(r, \theta, z, t, k) \in \mathcal{D}_{d} \times\left[t_{k}, t_{k+1}\right] \times \mathbb{N}^{*} \\
T\left(r, \theta, z, t_{k}, k\right)=\left\{\begin{array}{l}
T_{a} \\
T\left(r, \theta, z, t_{k}, k-1\right) \\
T\left(R_{m}, \theta, z, t_{\theta}^{k}, k\right)=T^{m}(\theta, z, k) \text { (measured conditions) }
\end{array} \quad\right. \text { (initial condition) }
\end{array}\right. \\
& \text { (Inverse part): }\left\{\begin{array}{l}
(r, \theta, z, t, k) \in \mathcal{D}_{i} \times\left[t_{k}, t_{k+1}\right] \times \mathbb{N}^{*} \\
T\left(r, \theta, z, t_{k}, k\right)=\left\{\begin{array}{ll}
T_{a} & k=1 \\
T\left(r, \theta, z, t_{k}, k-1\right) & k \geqslant 2
\end{array}\right. \text { (initial condition) }
\end{array}\right.
\end{aligned}
$$

Since the problem is linear, a well-known method is to find out an infinite family of solutions and to write a linear combination where the coefficients are identified with the initial condition of (5) and (6) and the measured conditions of (5). In this way, it is demonstrated (proof appended in Appendix A) that the function given by (7) is a solution of (2).

$$
\gamma J_{n}\left(r \sqrt{\frac{1}{D \tau}-\frac{i \omega n}{D}+\delta^{2}}\right) \exp (\operatorname{in} \theta) \exp (\delta z) \exp \left(-\frac{t}{\tau}\right)
$$

where $n$ is an integer, $\gamma, \tau$ and $\delta$ are complex numbers, and $J_{n}$ is the $n$th Bessel function of the first kind defined for a complex variable. It should be noted that the expression (7) remains a solution by taking $\delta=-\delta$ therefore the $\exp (\delta z)$ can also be replaced by $\cos (\delta z)$ or $\sin (\delta z)$.

\section{Decomposition}

It is very classic to divide the solution into two parts $T=T_{1}+T_{2}$. The first part $T_{1}$ is the steady solution for the $k$ th cycle and $T_{2}$ is the transient corrective solution, as done (among many others) by Hello and Vinet [26] who solved analytically a thermal problem related to cylindrical mirrors. 
The steady solution $T_{1}$ verifies the measured condition of (5) but takes not into account the initial condition of (5) and (6). This verification of the measured condition at the radius $R_{m}$ is seen for $T_{1}$ as a boundary condition specified on the sub-domain $\mathcal{D}_{d}$. The transient corrective solution $T_{2}$ verifies the initial condition of (5) and (6) minus the initial temperature field introduced by $T_{1}$ and vanishes at the radius $R_{m}$ (which is seen for $T_{2}$ as a boundary condition specified on the sub-domain $\mathcal{D}_{d}$ ). This decomposition is summarized in Figure 3.

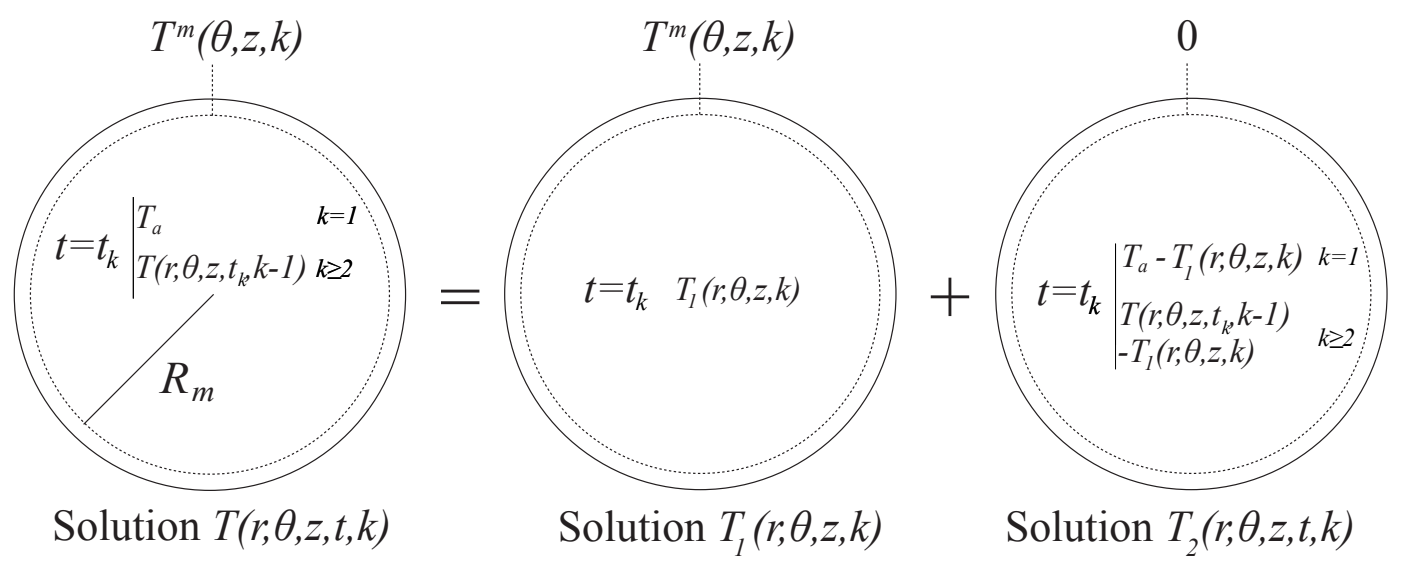

Figure 3: Superposition for each cycle

The analytical solution of the problem is found (solution of (2) in the form of (7) and verifying the conditions (5) and (6)), managing only one assumption due to the restriction of the measurement system (discussed in section 6). For each cycle $k$ :

$$
T(r, \theta, z, t, k)=T_{1}(r, \theta, z, k)+T_{2}(r, \theta, z, t, k)
$$

where $T_{1}$ is given by (16) and $T_{2}$ by (21).

All the components of the heat flux are obtained analytically by:

$$
\boldsymbol{H}(r, \theta, z, t, k)=\boldsymbol{H}_{1}(r, \theta, z, k)+\boldsymbol{H}_{2}(r, \theta, z, t, k)
$$

where $\boldsymbol{H}_{1}$ and $\boldsymbol{H}_{2}$ are respectively given by (18) and (31).

Therefore the outputs of the method (the temperature $T^{s}(\theta, z, t, k)$ or the heat flux $\boldsymbol{H}^{s}(\theta, z, t, k)$ at the outer radius, $s$ meaning surface) are given explicitly by replacing $r$ by $R_{s}$ in (8) or in (9). The procedure is summarized in Figure 4.

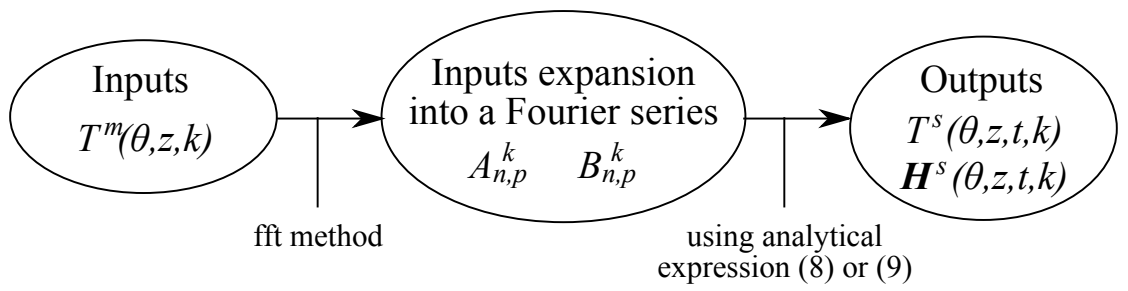

Figure 4: Procedure for each cycle

\section{Steady solution $T_{1}$ cycle by cycle}

\subsection{Measurement restrictions}

The solution $T$ is sought in the form $T=T_{1}+T_{2}$. The solution $T_{1}$ only has to match the measurements at the radius $R_{m}$ (i.e., verifying the partial boundary conditions of (5)), and $T_{2}$ only has to correct the solution $T_{1}$ in order to verify the initial condition of (5) and (6). If the temperature on the whole cylinder of radius $R_{m}$ (sub-domain $\mathcal{D}_{d}$ ) was known at any time, it would have been possible to expand the measurements into a Fourier series with time dependent coefficients. The identification of the time dependent Fourier coefficients as sums of 
time decreasing exponentials would allow to write the solution $T_{1}$ in the form of solutions given by (7). However, the measurement system being a unique line of local sensors (several sensors aligned along the axial direction) fixed into the body of the roll at the radius $R_{m}$, it provides only the temperatures at different angular positions at successive times. Therefore the time dependent expansion into a Fourier series of the temperature at the radius $R_{m}$ is impossible.

\subsection{Assumption: Time scale}

As it has been said, temperatures $T^{m}(\theta, z)$ are measured with respect to time, the measured condition in (3) is therefore incomplete. This difficulty is overcome by making the following assumption. It is assumed that during one cycle the variations of the temperature field are small. This assumption can be verified with numerical simulations of rolling process like the model proposed by Hacquin [11]. Therefore a natural characteristic time of the problem is the duration of one cycle. Thus, the time dependence of $T_{1}$ can be considered for increments of time of one cycle. The temporal evolution of the solution $T_{1}$ is only obtained from one cycle to another.

Because the solution $T_{1}(r, \theta, z, k)$ does not depend on time in the interval $\left[t_{k}, t_{k+1}\right]$, the family of solution can be restricted to:

$$
\gamma J_{n}\left(r \sqrt{-\frac{i \omega n}{D}+\delta^{2}}\right) \exp (\operatorname{in} \theta) \exp (\delta z)
$$

$T_{1}(r, \theta, z, k)$ is solution of (2) with the following measured conditions (it can be noted that these measured conditions are boundary condition (well defined) for $T_{1}$ on the sub-domain $\mathcal{D}_{d}$ ):

$$
\left\{\begin{array}{l}
(r, \theta, z, k) \in \mathcal{D}_{d} \times \mathbb{N}^{*} \\
T_{1}\left(R_{m}, \theta, z, k\right)=T^{m}(\theta, z, k) \text { (measured conditions) }
\end{array}\right.
$$

For each cycle, the measured temperature $T^{m}(\theta, z, k)$ is expanded into a double Fourier series, however since the axial heat flux vanishes at the edges (see conditions (3)), the form of the expansion is adapted to obtain automatically this condition. It should be noted that:

$$
\frac{d}{d z}\left[\cos \left(\frac{p \pi z}{L}\right)\right](z= \pm L)=0 \quad \text { and } \quad \frac{d}{d z}\left[\sin \left(\frac{(2 p+1) \pi z}{2 L}\right)\right](z= \pm L)=0
$$

Therefore the measured temperature are expanded as follows:

$$
T^{m}(\theta, z, k)=\sum_{n=-N_{1}}^{N_{1}} \sum_{p=0}^{P_{1}}\left[A_{n, p}^{k} \cos \left(\frac{p \pi z}{L}\right)+B_{n, p}^{k} \sin \left(\frac{(2 p+1) \pi z}{2 L}\right)\right] \exp (\operatorname{in} \theta)
$$

where $N_{1}$ and $P_{1}$ are two integers and $A_{n, p}^{k}$ and $B_{n, p}^{k}$ are the Fourier coefficients of the measurements done during the cycle $k$ :

$$
\begin{aligned}
& \forall n \in \mathbb{Z}, \forall p \geq 1\left\{\begin{array}{l}
A_{n, p}^{k}=\frac{1}{2 L \pi} \int_{-L}^{L} \int_{0}^{2 \pi} T^{m}(\theta, z, k) \exp (-i n \theta) \cos \left(\frac{p \pi z}{L}\right) \mathrm{d} \theta \mathrm{d} z \\
B_{n, p}^{k}=\frac{1}{2 L \pi} \int_{-L}^{L} \int_{0}^{2 \pi} T^{m}(\theta, z, k) \exp (-i n \theta) \sin \left(\frac{(2 p+1) \pi z}{2 L}\right) \mathrm{d} \theta \mathrm{d} z
\end{array}\right. \\
& \forall n \in \mathbb{Z} \\
& \left\{\begin{array}{l}
A_{n, 0}^{k}=\frac{1}{4 L \pi} \int_{-L}^{L} \int_{0}^{2 \pi} T^{m}(\theta, z, k) \exp (-i n \theta) \mathrm{d} \theta \mathrm{d} z \\
B_{n, 0}^{k}=\frac{1}{2 L \pi} \int_{-L}^{L} \int_{0}^{2 \pi} T^{m}(\theta, z, k) \exp (-i n \theta) \sin \left(\frac{\pi z}{2 L}\right) \mathrm{d} \theta \mathrm{d} z
\end{array}\right.
\end{aligned}
$$

The coefficients (14) can be computed classically from the measurements by using fast fourier transforms (fft). The algorithms are detailed in Appendix C. However (especially for the first few cycles), it can be noted that during a cycle the temperature field increases. Along the circumferential direction, at the end of the cycle, the measured temperature is higher than at the beginning even if the temperature is measured at the same angular position. Therefore the measured temperature $T^{m}(\theta, z, k)$ is not exactly $2 \pi$-periodic in $\theta$. Indeed, $T(r, \theta, z, t, k)$ is $2 \pi$-periodic at each time, but $T^{m}(\theta, z, k)=T\left(R_{m}, \theta, z, t_{\theta}^{k}, k\right)$ where $t_{\theta}^{k}=t_{k}+\theta / \omega$, thus for $\theta=0, t_{\theta}^{k}=t_{k}$ (being 
the time at the beginning of the cycle) and for $\theta=2 \pi, t_{\theta}^{k}=t_{k+1}$ (being the time at the end of the cycle). The discontinuity between the temperature at the end and at the beginning of each cycle creates a Gibb's effect in the expansion into a Fourier series (large oscillations near the discontinuity). This well-known effect can be reduced by applying a filter. Lanczos [27] and Acton [28, p.227] proposed a filter by multiplying by a gate function in the expansion:

$$
T^{m}(\theta, z, k) \simeq \sum_{n=-N_{1}}^{N_{1}} \sum_{p=0}^{P_{1}} \operatorname{sinc}^{g}\left(\frac{n}{N_{1}}\right)\left[A_{n, p}^{k} \cos \left(\frac{p \pi z}{L}\right)+B_{n, p}^{k} \sin \left(\frac{(2 p+1) \pi z}{2 L}\right)\right] \exp (\operatorname{in} \theta)
$$

where the power $g$ is a positive number (in the following $g=1$ or 0 ) and $\operatorname{sinc}(x)=\sin (\pi x) /(\pi x)$. The remaining oscillations (at the beginning and at the end) are simply removed from the signal. For each cycle, the function (16) is a solution of (2) in the form (7) and which matches the measurements at the radius $R_{m}$ (i.e., verifying boundary conditions (11)).

$$
T_{1}(r, \theta, z, k)=\sum_{n=-N_{1}}^{N_{1}} \sum_{p=0}^{P_{1}} \operatorname{sinc}^{g}\left(\frac{n}{N_{1}}\right)\left[A_{n, p}^{k} \frac{J_{n}\left(\zeta_{n, p} r\right)}{J_{n}\left(\zeta_{n, p} R_{m}\right)} \cos \left(\frac{\pi p z}{L}\right)+B_{n, p}^{k} \frac{J_{n}\left(\chi_{n, p} r\right)}{J_{n}\left(\chi_{n, p} R_{m}\right)} \sin \left(\frac{(2 p+1) \pi z}{2 L}\right)\right] \exp (\operatorname{in} \theta)
$$

where $\zeta_{n, p}$ and $\chi_{n, p}$ are defined by:

$$
\left\{\begin{array}{l}
\zeta_{n, p}=\sqrt{-\left(\frac{p \pi}{L}\right)^{2}-\frac{i \omega n}{D}} \\
\chi_{n, p}=\sqrt{-\left(\frac{(2 p+1) \pi}{2 L}\right)^{2}-\frac{i \omega n}{D}}
\end{array}\right.
$$

This part of the solution is exactly the solution of the steady heat equation, but updated at each cycle. It can be noted that the axial heat flux vanishes for $z= \pm L$ (because of (13)) as required in the set of conditions (3). The heat flux corresponding to $T_{1}$ is determined analytically by:

$$
\boldsymbol{H}_{1}(r, \theta, z, k)=\lambda\left(\frac{\partial T_{1}}{\partial r} \boldsymbol{e}_{r}+\frac{1}{r} \frac{\partial T_{1}}{\partial \theta} \boldsymbol{e}_{\theta}+\frac{\partial T_{1}}{\partial z} \boldsymbol{e}_{z}\right)
$$

where:

$$
\left\{\begin{array}{l}
\frac{\partial T_{1}}{\partial r}=\sum_{n=-N_{1}}^{N_{1}} \sum_{p=0}^{P_{1}} \operatorname{sinc}^{g}\left(\frac{n}{N_{1}}\right)\left[A_{n, p}^{k} \zeta_{n, p} \frac{J_{n}^{\prime}\left(\zeta_{n, p} r\right)}{J_{n}\left(\zeta_{n, p} R_{m}\right)} \cos \left(\frac{\pi p z}{L}\right)+B_{n, p}^{k} \chi_{n, p} \frac{J_{n}^{\prime}\left(\chi_{n, p} r\right)}{J_{n}\left(\chi_{n, p} R_{m}\right)} \sin \left(\frac{(2 p+1) \pi z}{2 L}\right)\right] \exp (\operatorname{in} \theta) \\
\frac{1}{r} \frac{\partial T_{1}}{\partial \theta}=\sum_{n=-N_{1}}^{N_{1}} \frac{i n}{r} \sum_{p=0}^{P_{1}} \operatorname{sinc}^{g}\left(\frac{n}{N_{1}}\right)\left[A_{n, p}^{k} \frac{J_{n}\left(\zeta_{n, p} r\right)}{J_{n}\left(\zeta_{n, p} R_{m}\right)} \cos \left(\frac{\pi p z}{L}\right)+B_{n, p}^{k} \frac{J_{n}\left(\chi_{n, p} r\right)}{J_{n}\left(\chi_{n, p} R_{m}\right)} \sin \left(\frac{(2 p+1) \pi z}{2 L}\right)\right] \exp (\operatorname{in} \theta) \\
\frac{\partial T_{1}}{\partial z}=\sum_{n=-N_{1}}^{N_{1}} \sum_{p=0}^{P_{1}} \operatorname{sinc}^{g}\left(\frac{n}{N_{1}}\right)\left[-\frac{\pi p A_{n, p}^{k}}{L} \frac{J_{n}\left(\zeta_{n, p} r\right)}{J_{n}\left(\zeta_{n, p} R_{m}\right)} \sin \left(\frac{\pi p z}{L}\right)+\frac{(2 p+1) \pi B_{n, p}^{k}}{2 L} \frac{J_{n}\left(\chi_{n, p} r\right)}{J_{n}\left(\chi_{n, p} R_{m}\right)} \cos \left(\frac{(2 p+1) \pi z}{2 L}\right)\right] \exp (\operatorname{in} \theta)
\end{array}\right.
$$

\subsection{Commentary and edge effect}

The radial variations of the temperature field are determined by $J_{n}\left(\zeta_{n, p} r\right)$ and $J_{n}\left(\chi_{n, p} r\right)$. Ratios $J_{n}\left(\zeta_{n, p} r\right) / J_{n}\left(\zeta_{n, p} R_{s}\right)$ and $J_{n}\left(\chi_{n, p} r\right) / J_{n}\left(\chi_{n, p} R_{s}\right)$ represent the attenuation (at the radius $r$ ) of the temperature signal considered at the surface of the roll. Indeed, if temperatures at the surface of the roll were known (which is not the case) and expanded into a Fourier series with Fourier coefficients denoted by $A_{n, p}^{s}$ and $B_{n, p}^{s}$ ( $s$ meaning surface) then the steady part of the temperature in the roll would be:

$$
\sum_{n=-N_{1}}^{N_{1}} \sum_{p=0}^{P_{1}}\left[A_{n, p}^{s} \frac{J_{n}\left(\zeta_{n, p} r\right)}{J_{n}\left(\zeta_{n, p} R_{s}\right)} \cos \left(\frac{\pi p z}{L}\right)+B_{n, p}^{s} \frac{J_{n}\left(\chi_{n, p} r\right)}{J_{n}\left(\chi_{n, p} R_{s}\right)} \sin \left(\frac{(2 p+1) \pi z}{2 L}\right)\right] \exp (\operatorname{in} \theta)
$$

It is therefore clear that scaling factors $J_{n}\left(\zeta_{n, p} r\right) / J_{n}\left(\zeta_{n, p} R_{s}\right)$ and $J_{n}\left(\chi_{n, p} r\right) / J_{n}\left(\chi_{n, p} R_{s}\right)$ are like a low-pass filter applied to the temperature signal at the surface of the roll. 
The figure 5 shows $J_{n}\left(\zeta_{n, p} r\right) / J_{n}\left(\zeta_{n, p} R_{s}\right)$ (coefficients $J_{n}\left(\chi_{n, p} r\right) / J_{n}\left(\chi_{n, p} R_{s}\right)$ are very similar) for some values of $n$ and $p$ against $r$ with $\omega=8 \pi(\mathrm{rad} / \mathrm{s})$ and $D=6 \times 10^{-6}$. A typical edge effect is demonstrated. The radius $R_{m}$ is chosen in order to be in the skin thickness of the roll. Beyond this skin thickness the measured signal would be almost constant (since ratios vanishes, corresponding Fourier coefficients would be discarded from the series expansion). It is obvious that the closer from the surface the sensor is fixed and the lower the attenuation level of the signal from the surface is, but ratios presented in figure 5 give precisely quantitative attenuation level as a function of the radius. It has been demonstrated by Weisz-Patrault et al. [19] and Legrand et al. [20] that it is possible to insert a thermocouple at around $0.5 \mathrm{~mm}$ from the surface of the roll, that is why this depth has been chosen in the following.

It should be noted that the $p$-dependence is almost negligible compared with the $n$-dependence (for $n \geq 1$ ). This is due to the fact that $(p \pi / L)^{2}<<\omega n / D$ and $((2 p+1) \pi /(2 L))^{2}<<\omega n / D$. Thus, even if the temperature signal at the surface is very heterogenous along the axial direction (corresponding to the $p$-dependence) 3D effects are rather limited since the attenuation of each Fourier coefficient does not significantly depend on $p$. This is why a 2D inverse method (that is to say $p=0$ ) gives for the reconstruction of surface temperature and heat flux good results as detailed in section 9. However, for $n=0$, coefficients $J_{0}\left(\zeta_{0, p} r\right) / J_{n}\left(\zeta_{0, p} R_{s}\right)$ and $J_{0}\left(\chi_{0, p} r\right) / J_{n}\left(\chi_{0, p} R_{s}\right)$ are $p$-dependent and do not present edge effect, as it can be seen in figure 6. Thus, for $n=0$ Fourier coefficients of the temperature signal at the surface of the roll are attenuated along the radial direction differently the ones from the others (according to $p$ ), especially in the roll core. This demonstrates a three-dimensional effect in the roll core, according to the radial and axial directions but independent on circumferential direction since $n=0$. These comments correspond to the analysis of Hacquin [11] who meshed the skin of the roll along the radial, circumferential and axial directions, and meshed the core of the roll along radial and axial directions only.

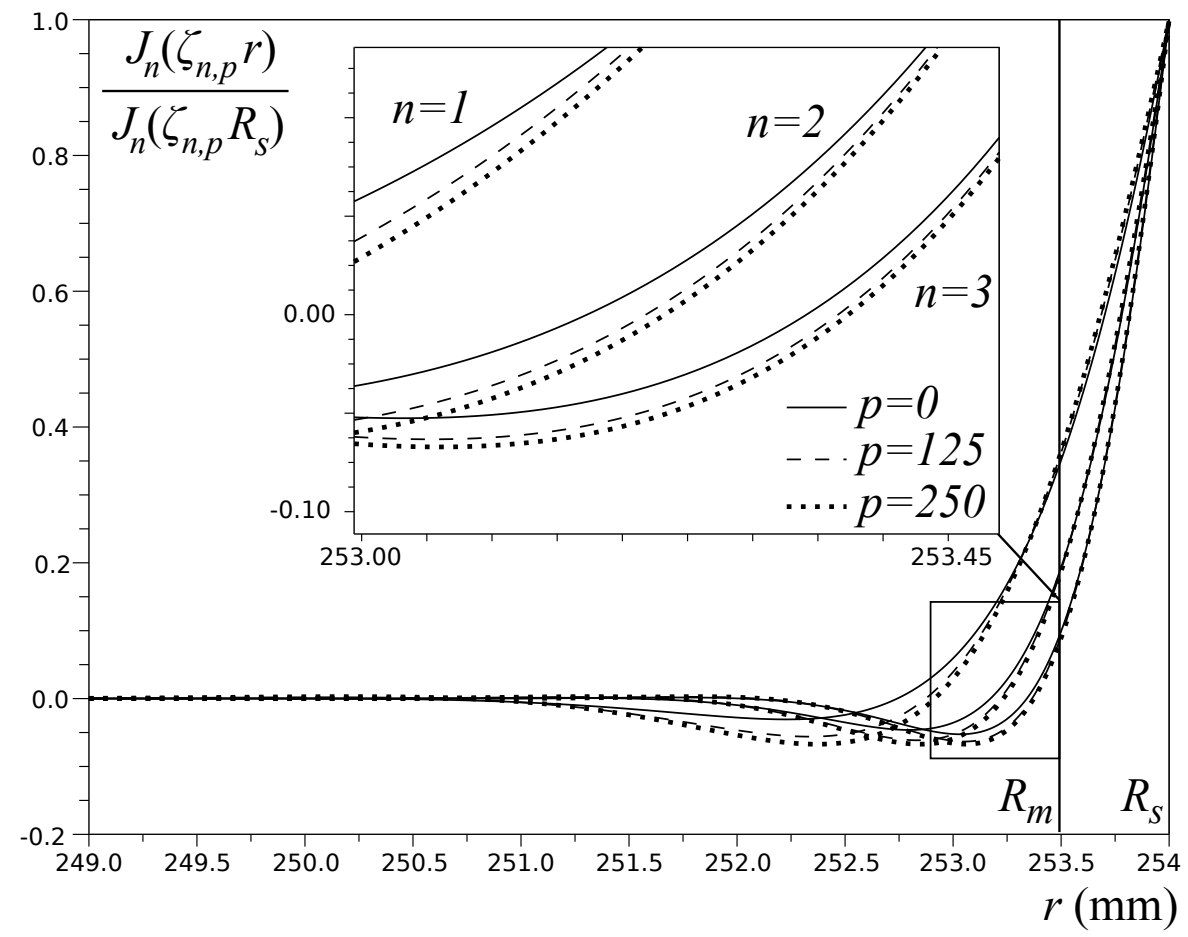

Figure 5: Edge effect 


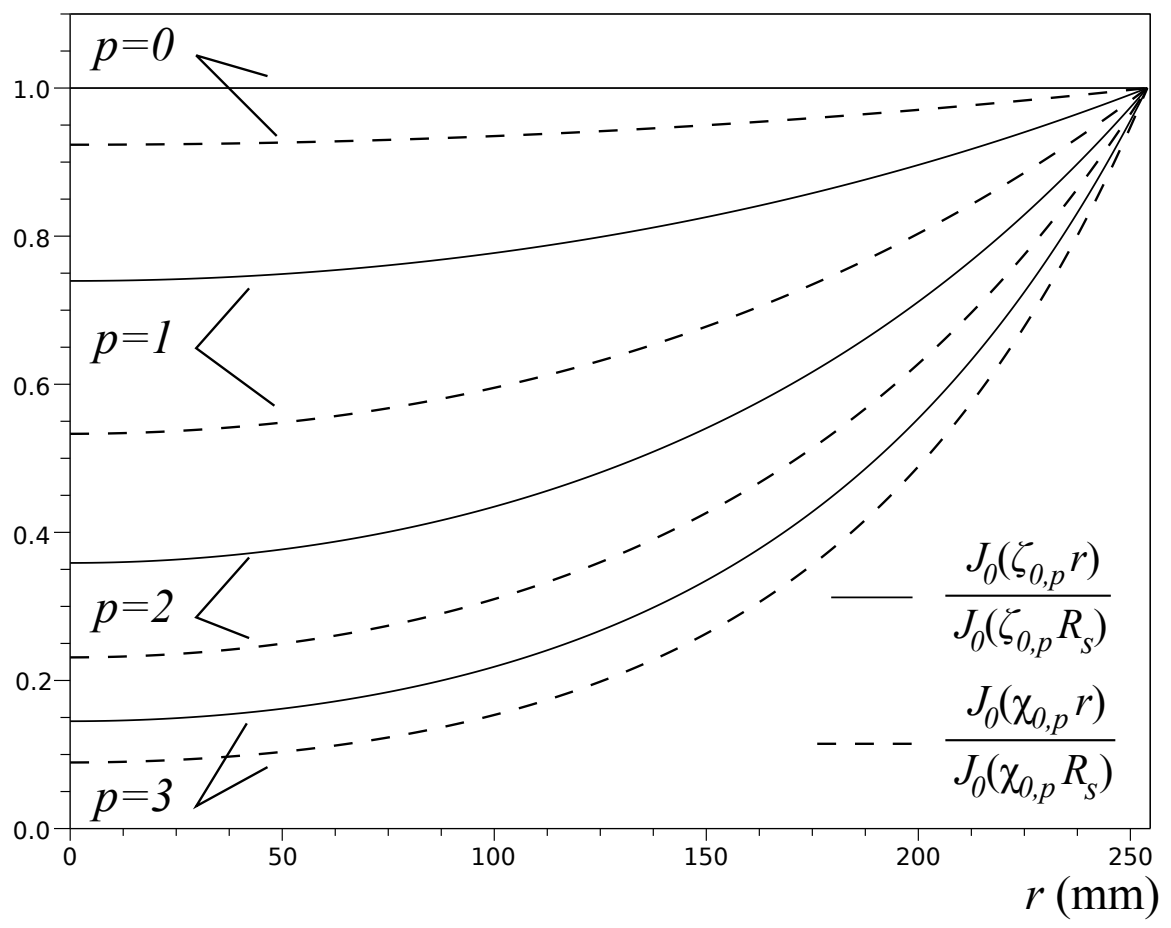

Figure 6: 3D effect in the core

\section{Transient solution $T_{2}$}

$T_{1}$ does not verify the initial condition (room temperature) of (5) and (6). A corrective solution $T_{2}$ is therefore needed. $T_{2}$ should verify the initial condition minus the initial temperature field introduced by the solution $T_{1}$. Moreover $T_{2}$ should vanish at the radius $R_{m}$, which is seen as a boundary condition on $\mathcal{D}_{d}$. Therefore, if $x_{n, q}$ are the positive zeros of the Bessel function of the order $n$ (arranged in ascending order of magnitude), then $T_{2}$ is sought in the form of Fourier-Bessel series (and so automatically vanishes at the radius $R_{m}$ ):

$T_{2}(r, \theta, z, t, k)=\sum_{n=-N_{1}}^{N_{1}} \sum_{p=0}^{P_{1}} \sum_{q=1}^{Q_{1}}\left[\alpha_{n, p, q}^{(k)} \exp \left(-\frac{t-t_{k}}{\tau_{n, p, q}}\right) \cos \left(\frac{p \pi z}{L}\right)+\beta_{n, p, q}^{(k)} \exp \left(-\frac{t-t_{k}}{v_{n, p, q}}\right) \sin \left(\frac{(2 p+1) \pi z}{2 L}\right)\right] J_{n}\left(x_{n, q} \frac{r}{R_{m}}\right) \exp (i n \theta)$

where $N_{2}$ is an integer, $\alpha_{n, p, q}^{(k)}$ and $\beta_{n, p, q}^{(k)}$ are complex numbers and the relaxation times $\tau_{n, p, q}$ and $v_{n, p, q}$ are given by:

$$
\left\{\begin{array}{l}
\tau_{n, p, q}=\frac{1}{D}\left(\left(\frac{x_{n, q}}{R_{m}}\right)^{2}+\left(\frac{p \pi}{L}\right)^{2}+\frac{i \omega n}{D}\right)^{-1} \\
v_{n, p, q}=\frac{1}{D}\left(\left(\frac{x_{n, q}}{R_{m}}\right)^{2}+\left(\frac{(2 p+1) \pi}{2 L}\right)^{2}+\frac{i \omega n}{D}\right)^{-1}
\end{array}\right.
$$

$T_{2}$ is written by considering each cycle as an independent problem. Thus, for the first cycle the initial condition is the room temperature. For the other cycles the initial condition is the temperature field at the end of the previous cycle. The initial condition of (5) and (6) can be written as follows (reminding that the time at the beginning of each cycle is $t_{k}$ ):

$$
\left\{\begin{array}{l}
\sum_{q=1}^{Q_{1}} \alpha_{n, p, q}^{(k)} J_{n}\left(x_{n, q} \frac{r}{R_{m}}\right)=u_{n, p}^{(k)}(r) \\
\sum_{q=1}^{Q_{1}} \beta_{n, p, q}^{(k)} J_{n}\left(x_{n, q} \frac{r}{R_{m}}\right)=v_{n, p}^{(k)}(r)
\end{array}\right.
$$


where:

$$
\begin{aligned}
& \begin{cases}u_{0,0}^{(k)}(r)=T_{a}-A_{0,0}^{k} & k=1 \\
u_{n, p}^{(k)}(r)=-\operatorname{sinc}^{g}\left(\frac{n}{N_{1}}\right) A_{n, p}^{k} \frac{J_{n}\left(\zeta_{n, p} r\right)}{J_{n}\left(\zeta_{n, p} R_{m}\right)} & k=1 \\
u_{n, p}^{(k)}(r)=\sum_{q=1}^{Q_{1}} \alpha_{n, p, q}^{(k-1)} \exp \left(-\frac{t_{k}-t_{k-1}}{\tau_{n, p, q}}\right) J_{n}\left(x_{n, q} \frac{r}{R_{m}}\right)+\operatorname{sinc}^{g}\left(\frac{n}{N_{1}}\right)\left(A_{n, p}^{k-1}-A_{n, p}^{k}\right) \frac{J_{n}\left(\zeta_{n, p} r\right)}{J_{n}\left(\zeta_{n, p} R_{m}\right)} & k \geqslant 2\end{cases} \\
& \begin{cases}v_{0,0}^{(k)}(r)=-B_{0,0}^{k} \frac{J_{0}\left(\chi_{0,0} r\right)}{J_{0}\left(\chi_{0,0} R_{m}\right)} & k=1 \\
v_{n, p}^{(k)}(r)=-\operatorname{sinc}^{g}\left(\frac{n}{N_{1}}\right) B_{n, p}^{k} \frac{J_{n}\left(\chi_{n, p} r\right)}{J_{n}\left(\chi_{n, p} R_{m}\right)} & k=1 \\
v_{n, p}^{(k)}(r)=\sum_{q=1}^{Q_{1}} \beta_{n, p, q}^{(k-1)} \exp \left(-\frac{t_{k}-t_{k-1}}{v_{n, p, q}}\right) J_{n}\left(x_{n, q} \frac{r}{R_{m}}\right)+\operatorname{sinc}^{g}\left(\frac{n}{N_{1}}\right)\left(B_{n, p}^{k-1}-B_{n, p}^{k}\right) \frac{J_{n}\left(\chi_{n, p} r\right)}{J_{n}\left(\chi_{n, p} R_{m}\right)} & k \geqslant 2\end{cases}
\end{aligned}
$$

It can be noted that for the $k$ th cycle $u_{n, p}^{(k)}(r)$ and $v_{n, p}^{(k)}(r)$ are functions of the variable $r$ known on the whole domain of variation of $r$ (i.e., $\left[0, R_{s}\right]$ ). Coefficients $\alpha_{n, p, q}^{(k)}$ and $\beta_{n, p, q}^{(k)}$ are given by (26) in an analytical form (called coefficients of Fourier-Bessel expansion) used by Sneddon [29, p.37] and previously demonstrated by Watson [30, pp.591594].

$$
\left\{\begin{array}{l}
\alpha_{n, p, q}^{(k)}=\frac{2}{R_{m}^{2} J_{n+1}^{2}\left(x_{n, q}\right)} \int_{0}^{R_{m}} \rho u_{n, p}^{(k)}(\rho) J_{n}\left(x_{n, q} \frac{\rho}{R_{m}}\right) \mathrm{d} \rho \\
\beta_{n, p, q}^{(k)}=\frac{2}{R_{m}^{2} J_{n+1}^{2}\left(x_{n, q}\right)} \int_{0}^{R_{m}} \rho v_{n, p}^{(k)}(\rho) J_{n}\left(x_{n, q} \frac{\rho}{R_{m}}\right) \mathrm{d} \rho
\end{array}\right.
$$

By using a well known result (27) given among others by Sneddon [29, p.27] and (28) given by Gradshteyn and Ryzhik [31] the expression of $\alpha_{n, p, q}^{(k)}$ and $\beta_{n, p, q}^{(k)}$ reduces to (29) and (30).

$$
\begin{gathered}
\int_{0}^{R_{m}} \rho J_{0}(x \rho) \mathrm{d} \rho=R_{m} \frac{J_{1}\left(R_{m} x\right)}{x} \\
\begin{cases}\int_{0}^{R_{m}} \rho J_{n}(x \rho) J_{n}(y \rho) \mathrm{d} \rho=R_{m} \frac{x J_{n+1}\left(R_{m} x\right) J_{n}\left(R_{m} y\right)-y J_{n+1}\left(R_{m} y\right) J_{n}\left(R_{m} x\right)}{x^{2}-y^{2}} & \\
\int_{0}^{R_{m}} \rho J_{n}(x \rho)^{2} \mathrm{~d} \rho=\frac{R_{m}^{2}}{2}\left(J_{n}\left(R_{m} x\right)^{2}-J_{n-1}\left(R_{m} x\right) J_{n+1}\left(R_{m} x\right)\right) & k=1 \\
\alpha_{n, p, q}^{(k)}=\frac{2}{J_{n+1}\left(x_{n, q}\right)} \frac{\operatorname{sinc}^{g}\left(n / N_{1}\right) A_{n, p}^{k}}{R_{m}^{2} \zeta_{n, p}^{2} x_{n, q}^{-1}-x_{n, q}} & k=1 \\
\alpha_{n, p, q}^{(k)}=-\alpha_{n, p, q}^{(k-1)} \exp \left(-\frac{t_{k}-t_{k-1}}{\tau_{n, p, q}}\right) \frac{J_{n-1}\left(x_{n, q}\right)}{J_{n+1}\left(x_{n, q}\right)}+\frac{2}{J_{n+1}\left(x_{n, q}\right)} \frac{\operatorname{sinc}^{g}\left(n / N_{1}\right)\left(A_{n, p}^{k}-A_{n, p}^{k-1}\right)}{R_{m}^{2} \zeta_{n, p}^{2} x_{n, q}^{-1}-x_{n, q}} \exp \left(\frac{t_{k}}{\tau_{n, p, q}}\right) & k \geqslant 2 \\
\beta_{0,0, q}^{(k)}=\frac{2}{J_{1}\left(x_{0, q}\right)} \frac{B_{0,0}^{2}}{R_{m}^{2} \chi_{0,0}^{2} x_{0, q}^{-1}-x_{0, q}} & k=1 \\
\beta_{n, p, q}^{(k)}=\frac{2}{J_{n+1}\left(x_{n, q}\right)} \frac{\operatorname{sinc}^{g}\left(n / N_{1}\right) B_{n, p}^{k}}{R_{m}^{2} \chi_{n, p}^{2} x_{n, q}^{-1}-x_{n, q}} & k=1 \\
\beta_{n, p, q}^{(k)}=-\beta_{n, p, q}^{(k-1)} \exp \left(-\frac{t_{k}-t_{k-1}^{k}}{v_{n, p, q}}\right) \frac{J_{n-1}\left(x_{n, q}\right)}{J_{n+1}\left(x_{n, q}\right)}+\frac{2}{J_{n+1}\left(x_{n, q}\right)} \frac{\operatorname{sinc}^{g}\left(n / N_{1}\right)\left(B_{n, p}^{k}-B_{n, p}^{k-1}\right)}{R_{m}^{2} \chi_{n, p}^{2} x_{n, q}^{-1}-x_{n, q}} \exp \left(\frac{t_{k}}{v_{n, p, q}}\right) & k \geqslant 2\end{cases}
\end{gathered}
$$


In their previous work Weisz-Patrault et al. [18] solved this kind of expansion into a Fourier-Bessel series by discretizing the problem along the radial direction which was very inelegant considering that an analytical solution exists.

The heat flux associated with $T_{2}$ is given by:

$$
\boldsymbol{H}_{2}(r, \theta, z, t, k)=\lambda\left(\frac{\partial T_{2}}{\partial r} \boldsymbol{e}_{r}+\frac{1}{r} \frac{\partial T_{2}}{\partial \theta} \boldsymbol{e}_{\theta}+\frac{\partial T_{2}}{\partial z} \boldsymbol{e}_{z}\right)
$$

where:

$$
\left\{\begin{array}{l}
\frac{T_{2}}{\partial r}=\sum_{n=-N_{1}}^{N_{1}} \sum_{p=0}^{P_{1}} \sum_{q=1}^{Q_{1}}\left[\alpha_{n, p, q}^{(k)} \exp \left(-\frac{t-t_{k}}{\tau_{n, p, q}}\right) \cos \left(\frac{p \pi z}{L}\right)+\beta_{n, p, q}^{(k)} \exp \left(-\frac{t-t_{k}}{v_{n, p, q}}\right) \sin \left(\frac{(2 p+1) \pi z}{2 L}\right)\right] \frac{x_{n, q}}{R_{m}} J_{n}^{\prime}\left(x_{n, q} \frac{r}{R_{m}}\right) \exp (\operatorname{in} \theta) \\
\frac{1}{r} \frac{T_{2}}{\partial \theta}=\sum_{n=-N_{1}}^{N_{1}} \frac{i n}{r} \sum_{p=0}^{P_{1}} \sum_{q=1}^{Q_{1}}\left[\alpha_{n, p, q}^{(k)} \exp \left(-\frac{t-t_{k}}{\tau_{n, p, q}}\right) \cos \left(\frac{p \pi z}{L}\right)+\beta_{n, p, q}^{(k)} \exp \left(-\frac{t-t_{k}}{v_{n, p, q}}\right) \sin \left(\frac{(2 p+1) \pi z}{2 L}\right)\right] J_{n}\left(x_{n, q} \frac{r}{R_{m}}\right) \exp (\operatorname{in} \theta) \\
\frac{T_{2}}{\partial z}=\sum_{n=-N_{1}}^{N_{1}} \sum_{p=0}^{P_{1}} \sum_{q=1}^{Q_{1}}\left[-\frac{p \pi \alpha_{n, p, q}^{(k)}}{L} \exp \left(-\frac{t-t_{k}}{\tau_{n, p, q}}\right) \sin \left(\frac{p \pi z}{L}\right)+\frac{(2 p+1) \pi \beta_{n, p, q}^{(k)}}{2 L} \exp \left(-\frac{t-t_{k}}{v_{n, p, q}}\right) \cos \left(\frac{(2 p+1) \pi z}{2 L}\right)\right] J_{n}\left(x_{n, q} \frac{r}{R_{m}}\right) \exp (\operatorname{in} \theta)
\end{array}\right.
$$

\section{Validation of the solution}

\subsection{Direct analytical temperature field}

The accuracy of the present inverse method and its noise sensitivity is demonstrated as follows. A prescribed time dependent temperature field (solution of (2)) is considered and called $T^{p}(r, \theta, z, t), p$ meaning prescribed. Therefore at the surface of the roll the temperature is $T^{p}\left(R_{s}, \theta, z, t\right)$. Then the temperature at the inner radius $R_{m}$ is extracted from the temperature field. The inputs are the temperatures at the radius $R_{m}$ (replacing measurements) and at the axial positions $z_{j}(j \in\{1 ; \ldots ; S\}$, where $S$ is the number of thermocouples) and considered for successive times (called $\left.t_{\theta}^{k}=t_{k}+\theta / \omega\right)$ according to the rotation speed of the roll and the index $k$ of the cycle: $T^{m}\left(\theta, z_{j}, k\right)=$ $T^{p}\left(R_{m}, \theta, z_{j}, t_{\theta}^{k}\right)$. Then cubic splines are used to interpolate the input signal along both the circumferential and axial directions, thus $T^{m}(\theta, z, k)$ is obtained. The outputs are then calculated and compared to the prescribed temperature at the surface of the roll. The error $\epsilon$ given in (33) is used as a percentage to evaluate the quality of the reconstruction.

$$
\epsilon=100 \sqrt{\frac{\int_{-L}^{L} \int_{0}^{2 \pi}\left[T^{s}\left(\theta, z, t_{\theta}^{k}, k\right)-T^{p}\left(R_{s}, \theta, z, t_{\theta}^{k}\right)\right]^{2} \mathrm{~d} \theta \mathrm{d} z}{\int_{-L}^{L} \int_{0}^{2 \pi}\left[T^{p}\left(R_{s}, \theta, z, t_{\theta}^{k}\right)\right]^{2} \mathrm{~d} \theta \mathrm{d} z}}
$$

The prescribed temperature field is:

$$
\begin{array}{r}
T^{p}(r, \theta, z, t)=\sum_{n=-N_{2}}^{N_{2}} \sum_{p=0}^{P_{2}}\left[a_{n, p} J_{n}\left(\zeta_{n, p} r\right) \cos \left(\frac{\pi p z}{L}\right)+b_{n, p} J_{n}\left(\chi_{n, p} r\right) \sin \left(\frac{\pi(2 p+1) z}{2 L}\right)\right] \exp (\text { in } \theta) \\
+\sum_{n=-N_{2}}^{N_{2}} \sum_{p=0}^{P_{2}} \sum_{q=1}^{Q_{2}} c_{n, p, q} J_{n}\left(r \sqrt{\frac{1}{D \tau_{n, p, q}^{*}}-\frac{i \omega n}{D}-\left(\frac{\pi p}{L}\right)^{2}}\right) \exp \left(\frac{-t}{\tau_{n, p, q}^{*}}\right) \exp (\operatorname{in} \theta) \cos \left(\frac{\pi p z}{L}\right) \\
+\sum_{n=-N_{2}}^{N_{2}} \sum_{p=0}^{P_{2}} \sum_{q=1}^{Q_{2}} d_{n, p, q} J_{n}\left(r \sqrt{\frac{1}{D v_{n, p, q}^{*}}-\frac{i \omega n}{D}-\left(\frac{\pi(2 p+1)}{2 L}\right)^{2}}\right) \exp \left(\frac{-t}{v_{n, p, q}^{*}}\right) \exp (\operatorname{in} \theta) \sin \left(\frac{\pi(2 p+1) z}{2 L}\right)
\end{array}
$$

where $y_{n, q}$ are the successive positive zeros of the functions $y \mapsto h_{n}(y)=\lambda\left(y / R_{s}\right) J_{n}^{\prime}(y)+\mathrm{HTC} \times J_{n}(y)$ and $a_{n, p}$ and $b_{n, p}$ are complex numbers given by (B.9), $c_{n, p, q}$ and $d_{n, p, q}$ are complex number given by (B.13), $\tau_{n, p, q}^{*}$ and $v_{n, p, q}^{*}$ are given by (B.8), and $N_{2}, P_{2}$ and $Q_{2}$ are integers.

The temperature field $T^{p}(r, \theta, z, t)$ given by (34) is in the form of (7) and is therefore an exact solution of the governing equation (2). Coefficients $a_{n, p}, b_{n, q}, c_{n, p, q}$ and $d_{n, p, q}$ are chosen such as the temperature field $T^{p}(r, \theta, z, t)$ corresponds to a roll surrounded by the ambient temperature $T_{a}$ everywhere but in a part $(\theta, z) \in[\pi-\Theta, \pi+\Theta] \times$ $[-L+Z, L-Z]$ where the surrounding temperature is a slope $T^{*}(\theta, z)$ as defined in Figure B.27 (simulating the contact between the strip and the roll). The heat flux entering the roll is defined classically by a heat transfer coefficient HTC. A constant HTC has been considered for simplicity (HTC $=7 \times 10^{-4} \mathrm{~W} \cdot \mathrm{m}^{-2} \cdot \mathrm{K}^{-1}$ ), because this choice make the problem linear, a time or angular dependent HTC would not enable an analytical solution. 
This value of HTC corresponds to the contact between the roll and the strip (steel/steel) but is physically strongly incorrect outside the contact interval (air/steel or water/steel). Therefore these boundary conditions are very simple compared with real boundary conditions occurring during rolling processes, but for the purpose of validating the present inverse method and its noise sensitivity, they are sufficiently severe boundary conditions because $\Theta$ and $T^{*}(\theta, z)$ are adjusted to have a sharp temperature peak quite close from results classically obtained with predictive models of section 1.1. Details concerning the calculation of $a_{n, p}, b_{n, q}, c_{n, p, q}$ and $d_{n, p, q}$ are appended in Appendix B. The surrounding temperature $T^{*}(\theta, z)$ is chosen strongly heterogeneous along the axial direction (near strip edges) to show the accuracy of the present method.

\subsection{Discussion of parameters}

The parameters of the problem are listed in Table 2. The rotation speed is settled to $8 \pi(\mathrm{rad} / \mathrm{s})$ or $6.4 \mathrm{~m} / \mathrm{s}$ considering the radius of the roll, which is the kind of speed commonly used for industrial hot rolling conditions. The angular part $\Theta$ where the roll is heated for the validation of the inverse solution presented in this paper corresponds approximately to hot rolling conditions with large reductions. The half-length of the roll $L$ is consistent with industrial mills. The order $g$ of the filter introduced in (15), is settled to 1 for the first cycle as proposed by Acton [28, p.227], for the cycle after 10 minutes, there is no discontinuity of measured temperatures between the beginning and the end of the cycle (as it can been seen in figures 11(b) and 12(b)), then $g=0$ so that the filter is removed from the development.

Integers $N_{2}, P_{2}$ and $Q_{2}$ are the truncation numbers for the prescribed temperature field. Low values for $\theta$ and $z$ dependences $\left(N_{2}\right.$ and $\left.P_{2}\right)$ have been tested for simplicity, since any values give an analytical solution of the heat equation. On the other hand $Q_{2}$ determines the quality of how the initial condition is verified, therefore $Q_{2}$ is settled to a rather large value (as well as $Q_{1}$ for the inverse solution) so that a good convergence of corresponding Fourier-Bessel series is ensured.

\begin{tabular}{|ll|c|}
\hline \multicolumn{2}{|c|}{ Parameter } & Value \\
\hline$N_{1}$ & $(-)$ & 50 \\
\hline$P_{1}$ & $(-)$ & 50 \\
\hline$Q_{1}$ & $(-)$ & 200 \\
\hline$N_{2}$ & $(-)$ & 20 \\
\hline$P_{2}$ & $(-)$ & 14 \\
\hline$Q_{2}$ & $(-)$ & 200 \\
\hline$N_{\theta}$ & $(-)$ & 100 \\
\hline$N_{z}$ & $(-)$ & 30 \\
\hline$S$ & $(-)$ & 30 \\
\hline$R_{S}$ & $(\mathrm{~m})$ & 0.254 \\
\hline$R_{m}$ & $(\mathrm{~m})$ & 0.2535 \\
\hline$D$ & $\left(\mathrm{~m}^{2} / \mathrm{s}\right)$ & $6 \times 10^{-6}$ \\
\hline$\lambda$ & $\left(\mathrm{W} \cdot \mathrm{m}^{-1} \cdot \mathrm{K}^{-1}\right)$ & 52 \\
\hline$\omega$ & $(\mathrm{rad} / \mathrm{s})$ & $8 \pi$ \\
\hline$f$ & $(\mathrm{~Hz})$ & 1000 \\
\hline$T_{a}$ & $(\mathrm{~K})$ & 293.15 \\
\hline $\mathrm{HTC}$ & $\left(\mathrm{W} \cdot \mathrm{m}^{-2} \cdot \mathrm{K}^{-1}\right)$ & $7 \times 10^{4}$ \\
\hline$\Theta$ & $(\mathrm{rad})$ & $\pi / 10$ \\
\hline$L$ & $(\mathrm{~mm})$ & 700 \\
\hline$Z$ & $(\mathrm{~mm})$ & 200 \\
\hline$g$ & $(-)$ & $0 / 1$ \\
\hline
\end{tabular}

Table 2: Computing values

For the inverse solution the truncation numbers $N_{1}$ and $P_{1}$ are determined as follows. The Fourier coefficients $A_{n, p}^{k}$ and $B_{n, p}^{k}$ are amplified by $J_{n}\left(\zeta_{n, p} R_{s}\right) / J_{n}\left(\zeta_{n, p} R_{m}\right)$ and $J_{n}\left(\chi_{n, p} R_{s}\right) / J_{n}\left(\chi_{n, p} R_{m}\right)$. Thus, small errors for the computation of $A_{n, p}^{k}$ and $B_{n, p}^{k}$ are more and more amplified when $n$ and $p$ increase, until that the solution diverge. In Figure 7, coefficients $J_{n}\left(\zeta_{n, p} R_{s}\right) / J_{n}\left(\zeta_{n, p} R_{m}\right)$ are plotted against $n$ for some values of $p$ (similar figure could be obtained for $\left.J_{n}\left(\chi_{n, p} R_{s}\right) / J_{n}\left(\chi_{n, p} R_{m}\right)\right)$. Sharp variations can be noted according to $n$ when coefficients seem almost 
independent on $p$. That is why $P_{1}$ is simply taken such as the sum has converged: it is not significant to be very precise because coefficients $J_{n}\left(\zeta_{n, p} R_{s}\right) / J_{n}\left(\zeta_{n, p} R_{m}\right)$ diverge very slowly according to $p$. The choice of $P_{1}$ is shown in Figure 8. For $N_{1}$, since coefficients $J_{n}\left(\zeta_{n, p} R_{s}\right) / J_{n}\left(\zeta_{n, p} R_{m}\right)$ and $J_{n}\left(\chi_{n, p} R_{s}\right) / J_{n}\left(\chi_{n, p} R_{m}\right)$ strongly diverge when $n$ increases, errors on $A_{n, p}^{k}$ and $B_{n, p}^{k}$ are rapidly amplified until that the solution diverge. Thus, $N_{1}$ should be taken such as errors are not amplified to much as demonstrated in Figures 9 and 10. Usually, when noise is added to the inputs, errors made on Fourier coefficients $A_{n, p}^{k}$ and $B_{n, p}^{k}$ increase and $N_{1}$ should be reduced. However, the particular case, that is proposed in this paper in order to validate the method, is simple enough to keep the same value of $N_{1}$ because as it can been seen in Figure 7 amplification factors begin to diverge for values of $n \geq N_{1}=50$.

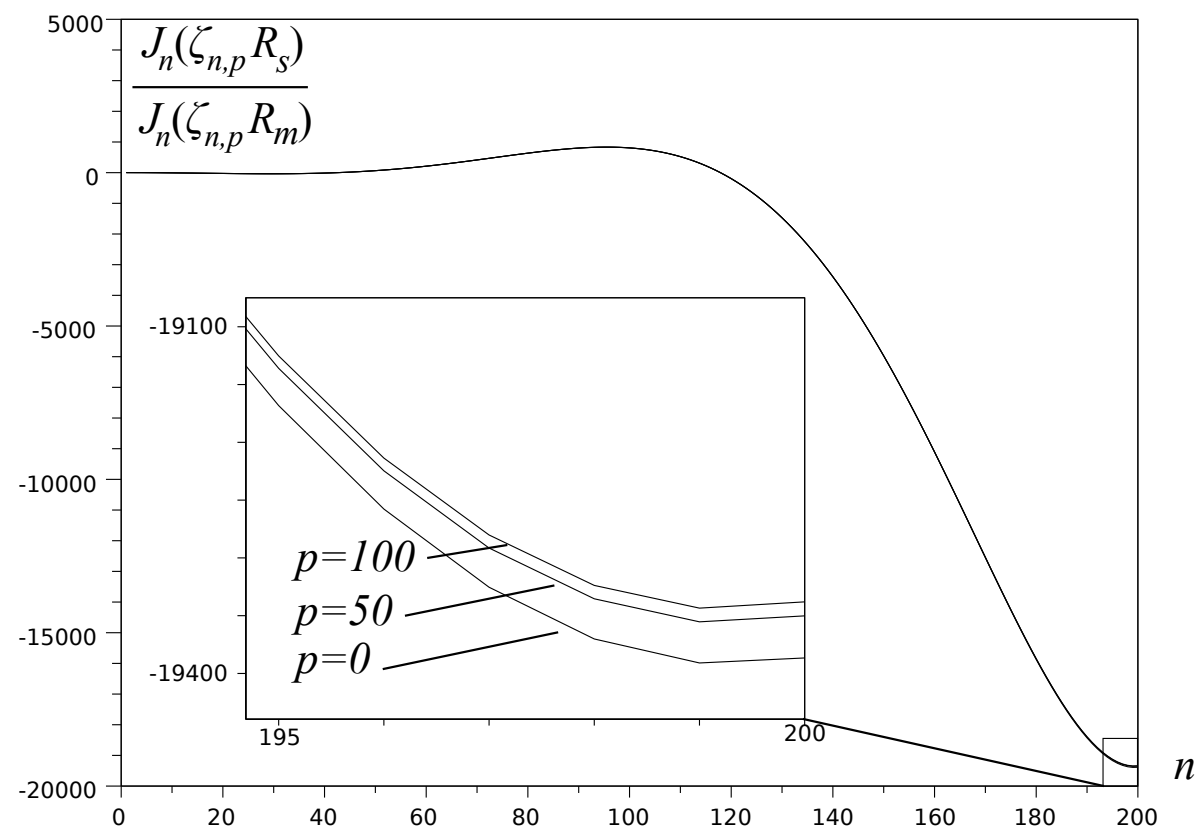

Figure 7: $J_{n}\left(\zeta_{n, p} R_{s}\right) / J_{n}\left(\zeta_{n, p} R_{m}\right)$ vs $n$ for some values of $p$

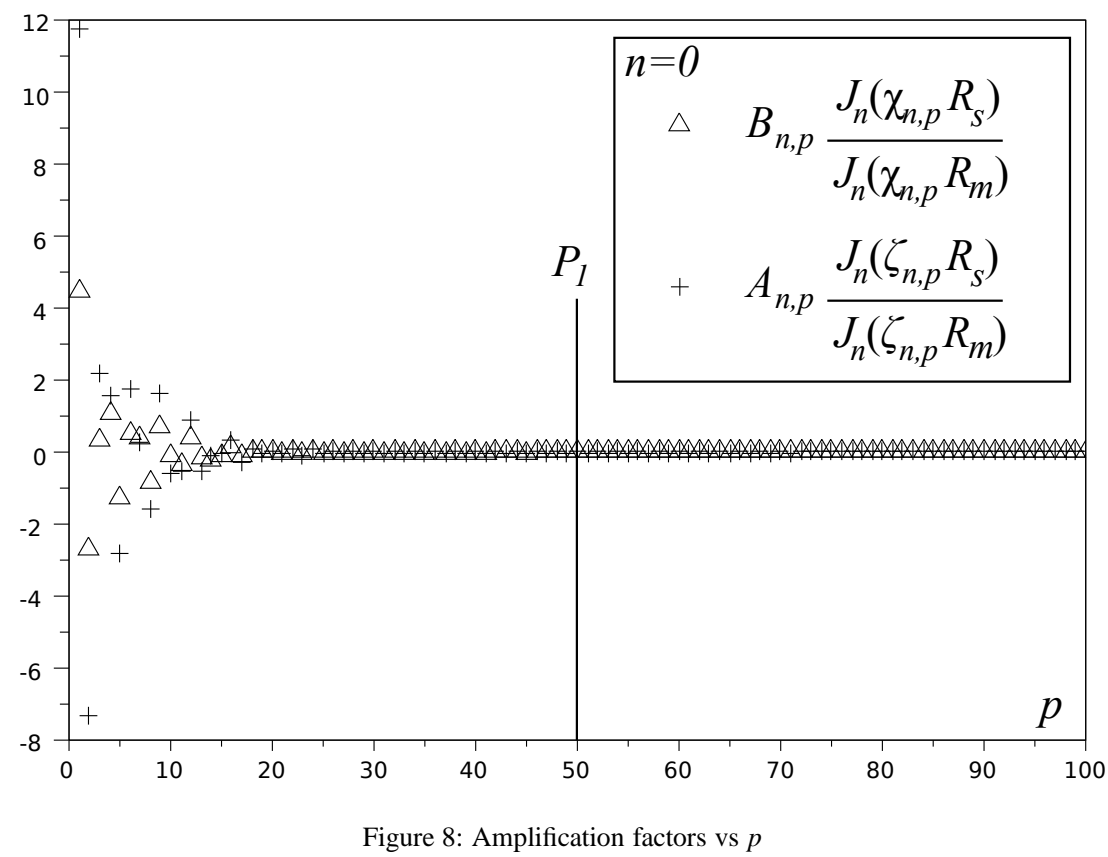




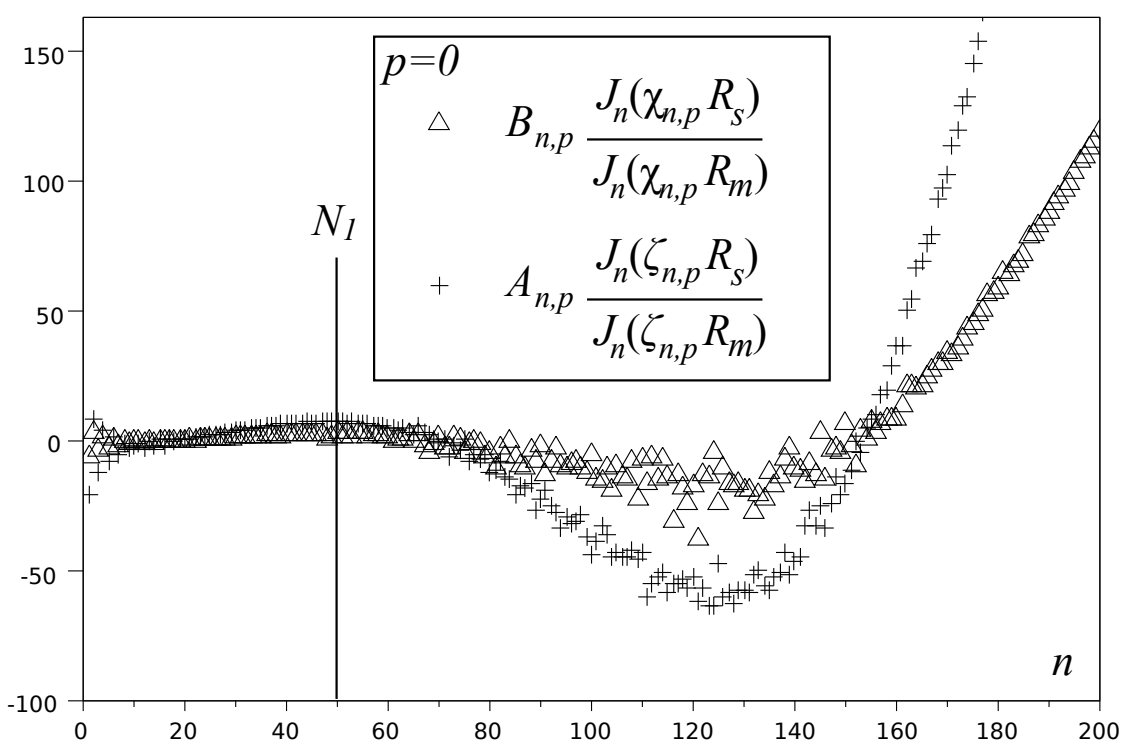

Figure 9: Amplification factors vs $n$ for $p=0$

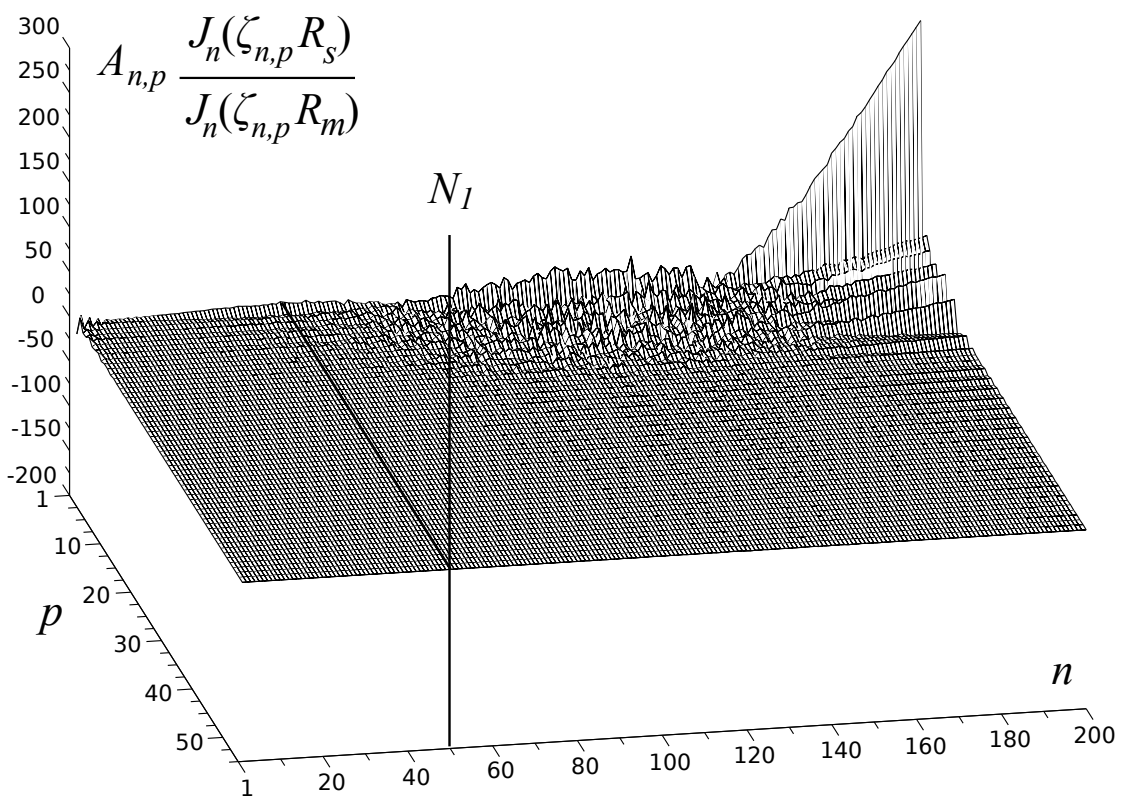

Figure 10: Amplification factors vs $n$ and $p$

\subsection{Reconstruction free from noise}

Using (34), the inputs are produced for two different cycles: the first cycle and the cycle after 10 minutes. The prescribed temperatures at the surface of the roll are given in Figure 11, and the temperatures at the inner radius $R_{m}$ (replacing measurements) are given in Figure 12. In order to compute coefficients $A_{n, p}^{k}$ and $B_{n, p}^{k}$ given by (14), fft are used as detailed in Appendix C. Input signal is interpolated with cubic splines. $N_{z}^{i}$ and $N_{\theta}^{i}$ denote the numbers of interpolation points. A compromise between accuracy and short computation times should be found. Two sets of parameters are tested: $N_{z}^{i}=100$ and $N_{\theta}^{i}=1000$ leading to a CPU time of $0.07 \mathrm{~s} / \mathrm{cycle}$ and $N_{z}^{i}=1000$ and $N_{\theta}^{i}=1000$ leading to a CPU time of $0.5 \mathrm{~s} /$ cycle. This has to be seen as a rule of thumb, no specific study about the solution quality considering the number of interpolation points has been done. The outputs of the method compared with the prescribed temperature at the surface of the roll are given in Figures 13 and 17 for the first cycle and in Figures 15 and 19 for the cycle after 10 minutes. The quantified errors are listed 
in Table 3. Excellent accuracy is observed in both cases, but accuracy is better near strip edges for the second set of parameters. Figures of the absolute difference between the reconstructed temperature and the prescribed temperature (error of reconstruction expressed in Kelvin) are also given for emphasizing the reconstruction quality near strip edges (see Fig.14, 16, 18 and 20).

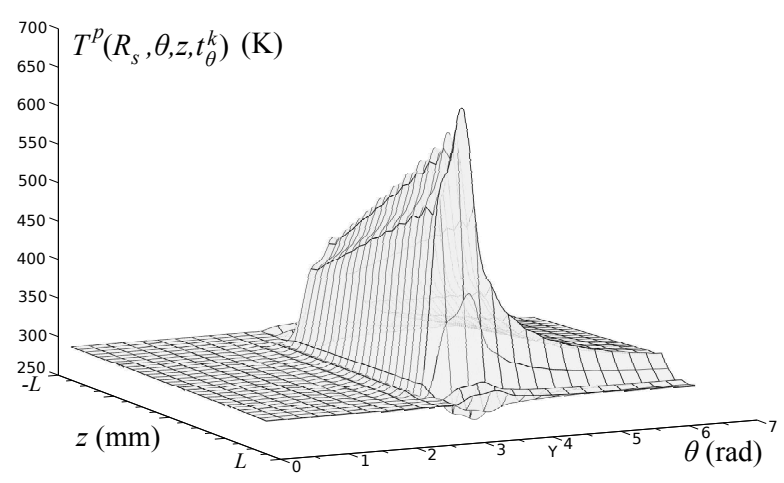

(a) First cycle

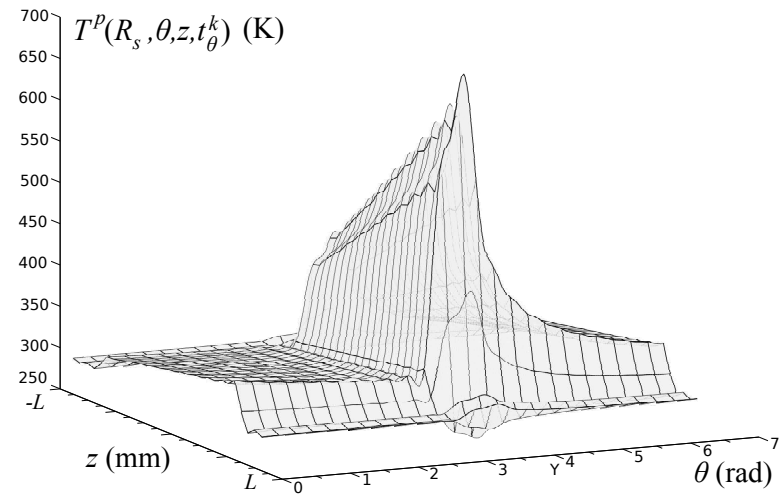

(b) Cycle after $10 \mathrm{~min}$

Figure 11: Prescribed temperatures at the surface of the roll

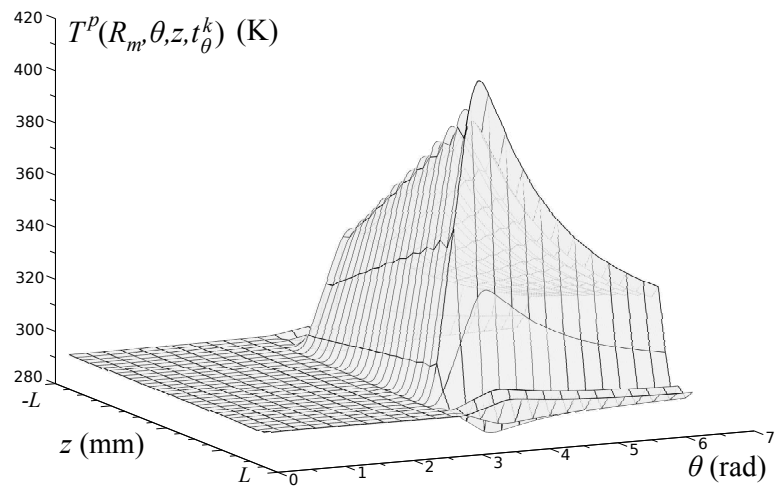

(a) First cycle

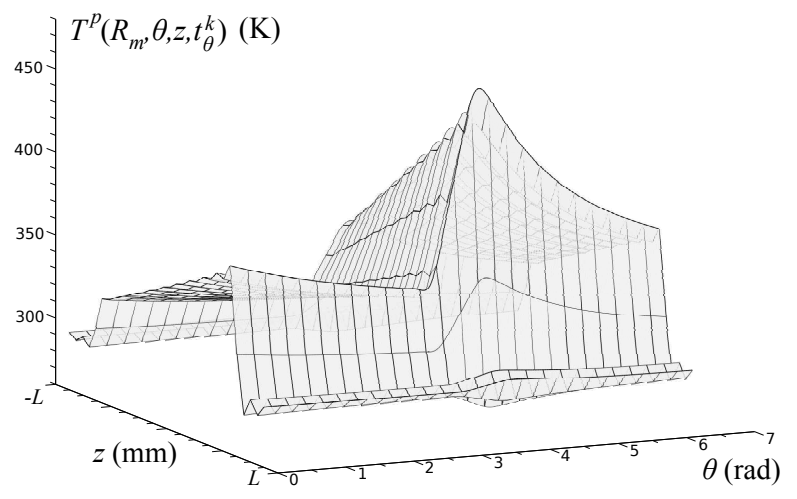

(b) Cycle after $10 \mathrm{~min}$

Figure 12: Prescribed temperatures (replacing measurements) at the inner radius $R_{m}$ 


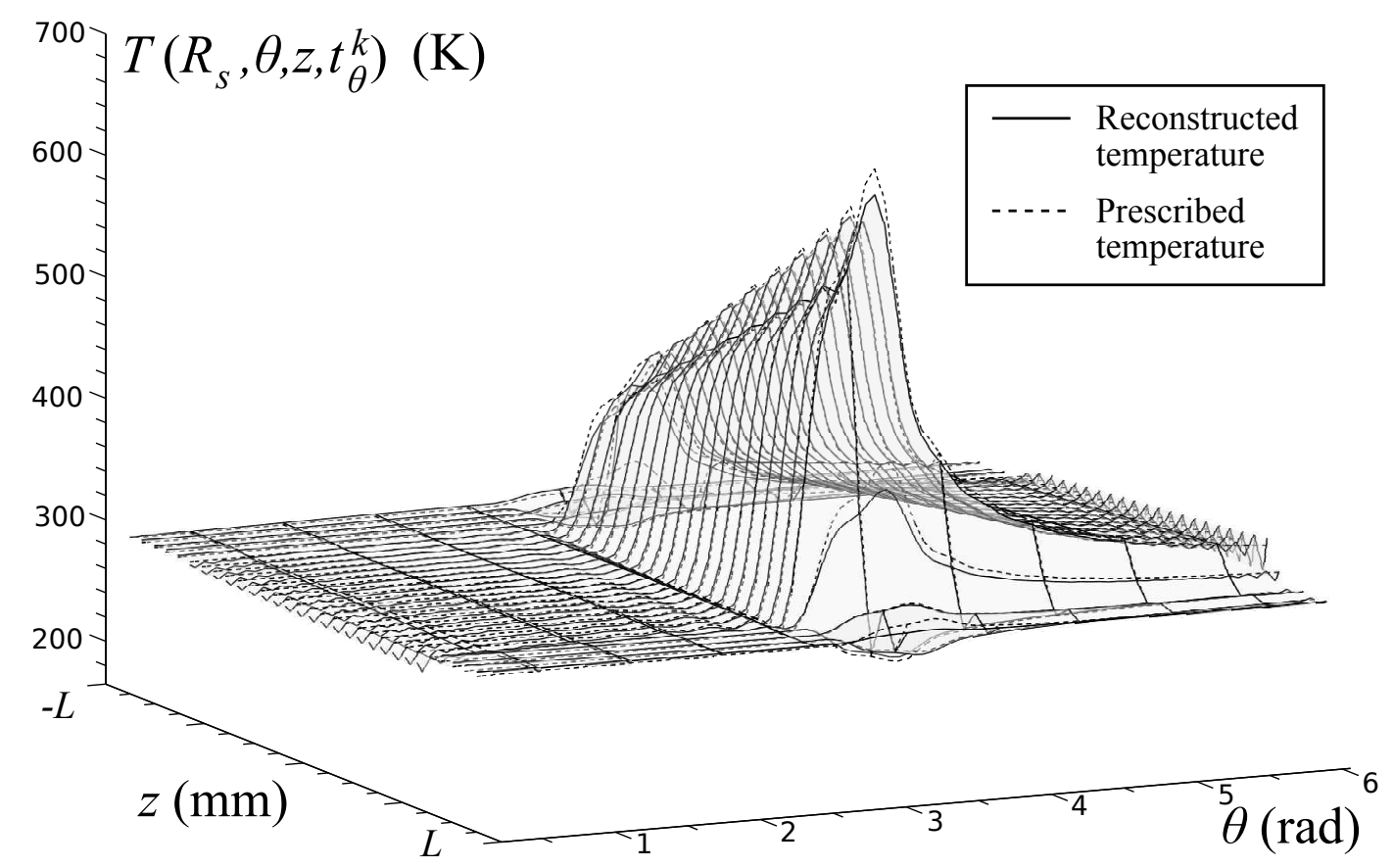

Figure 13: First cycle, reconstructed temperatures at the surface of the roll without noise for $N_{z}^{i}=100$ and $N_{\theta}^{i}=1000$

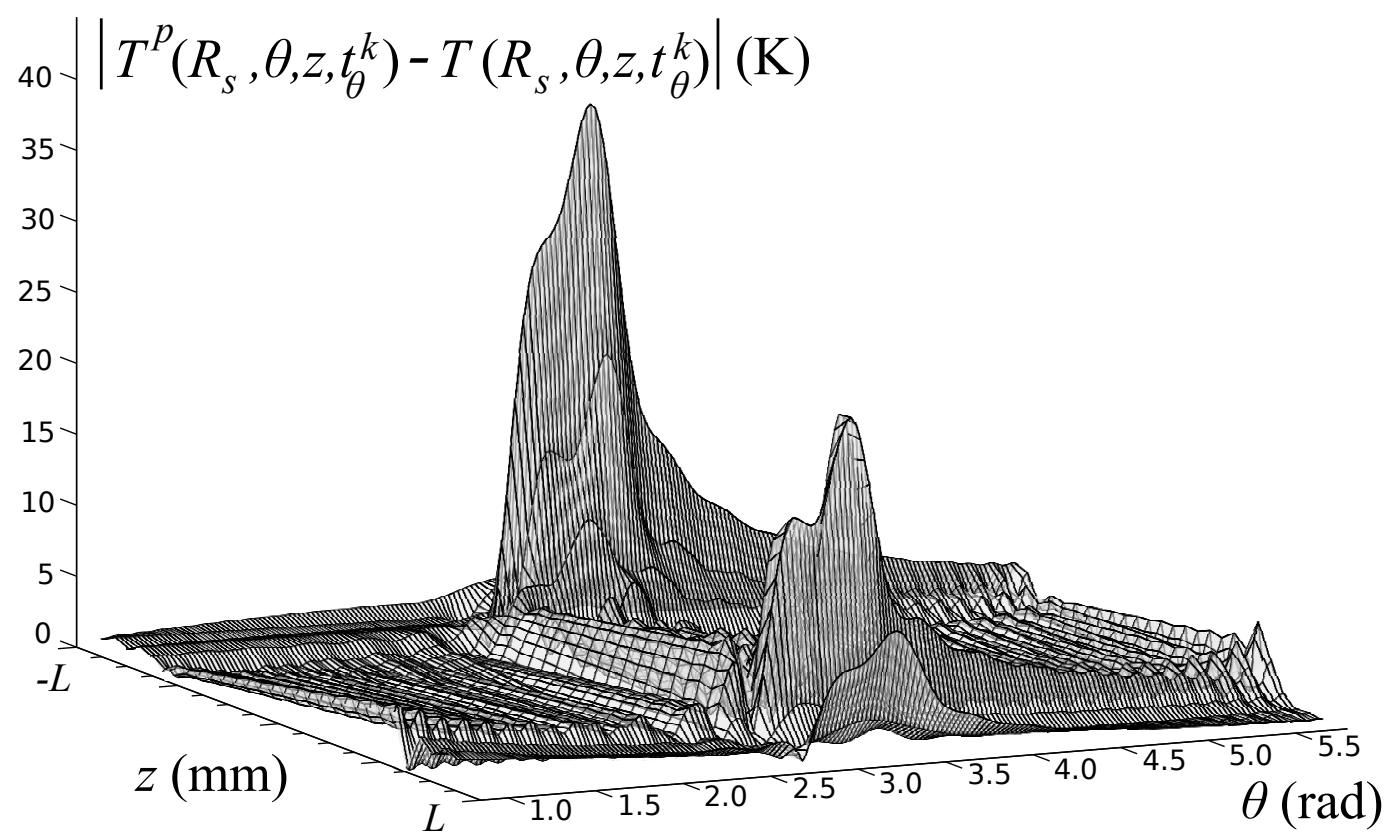

Figure 14: First cycle, error of reconstruction without noise for $N_{z}^{i}=100$ and $N_{\theta}^{i}=1000$ 


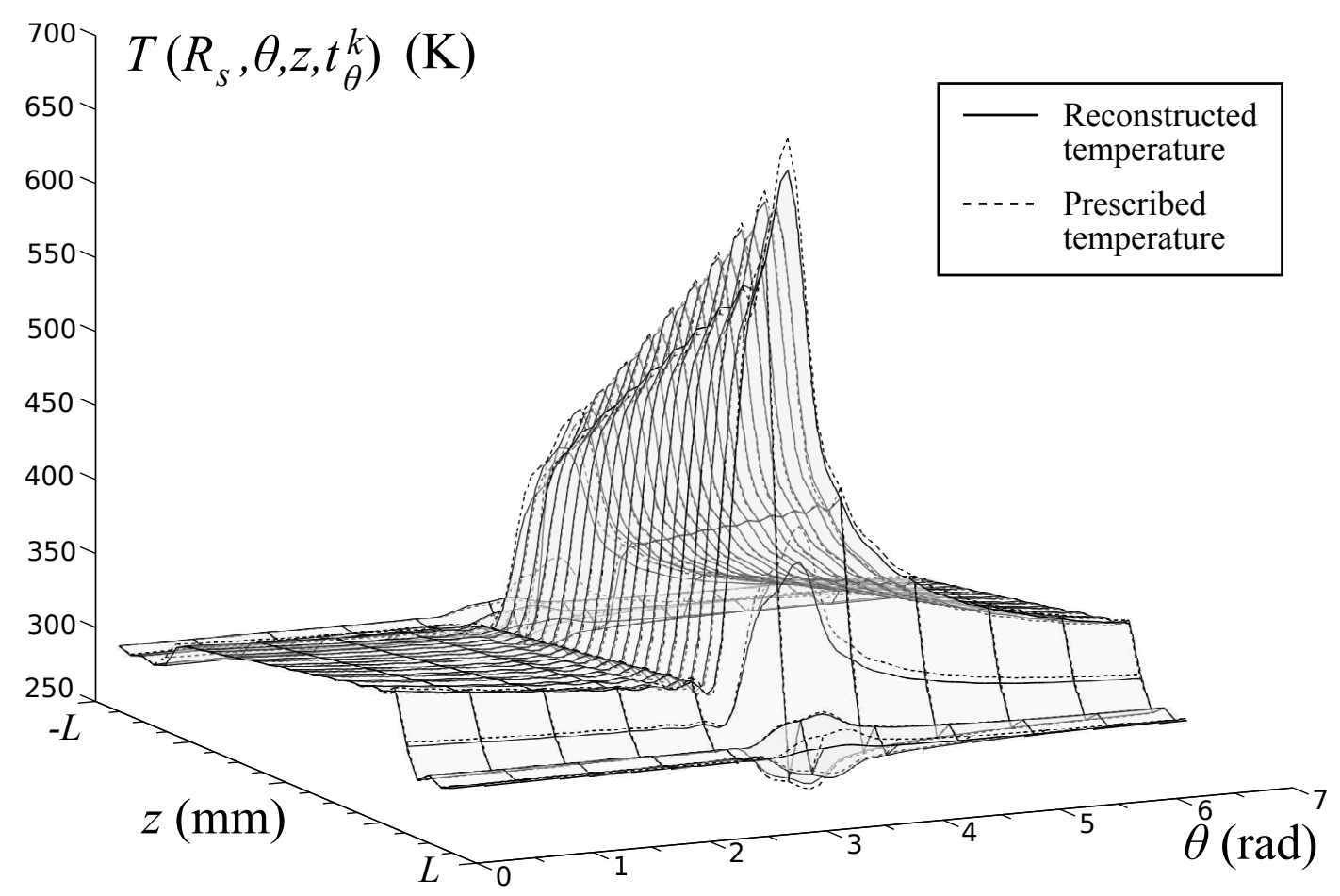

Figure 15: Cycle after $10 \mathrm{~min}$, reconstructed temperatures at the surface of the roll without noise for $N_{z}^{i}=100$ and $N_{\theta}^{i}=1000$

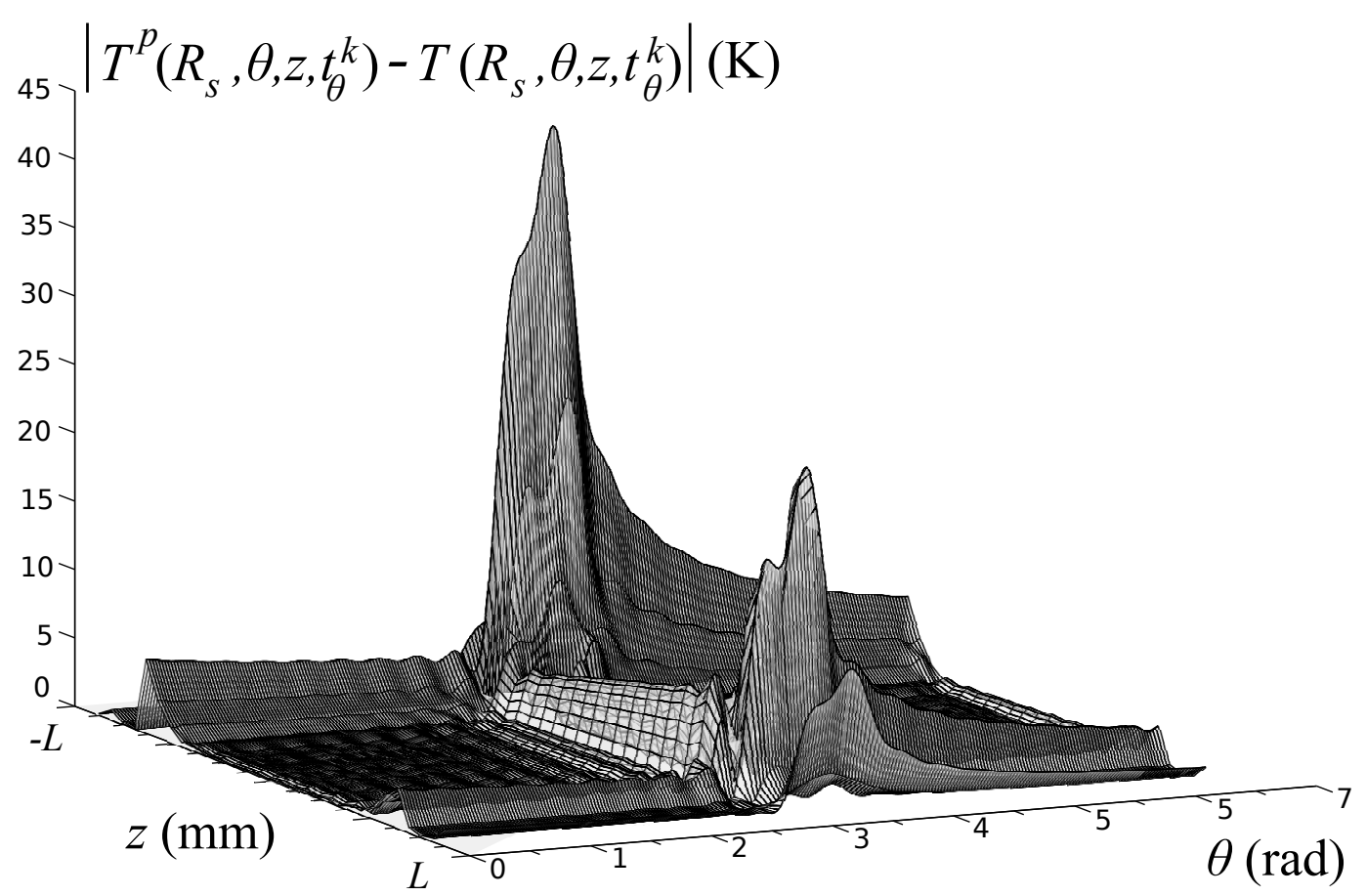

Figure 16: Cycle after $10 \mathrm{~min}$, error of reconstruction without noise for $N_{z}^{i}=100$ and $N_{\theta}^{i}=1000$ 


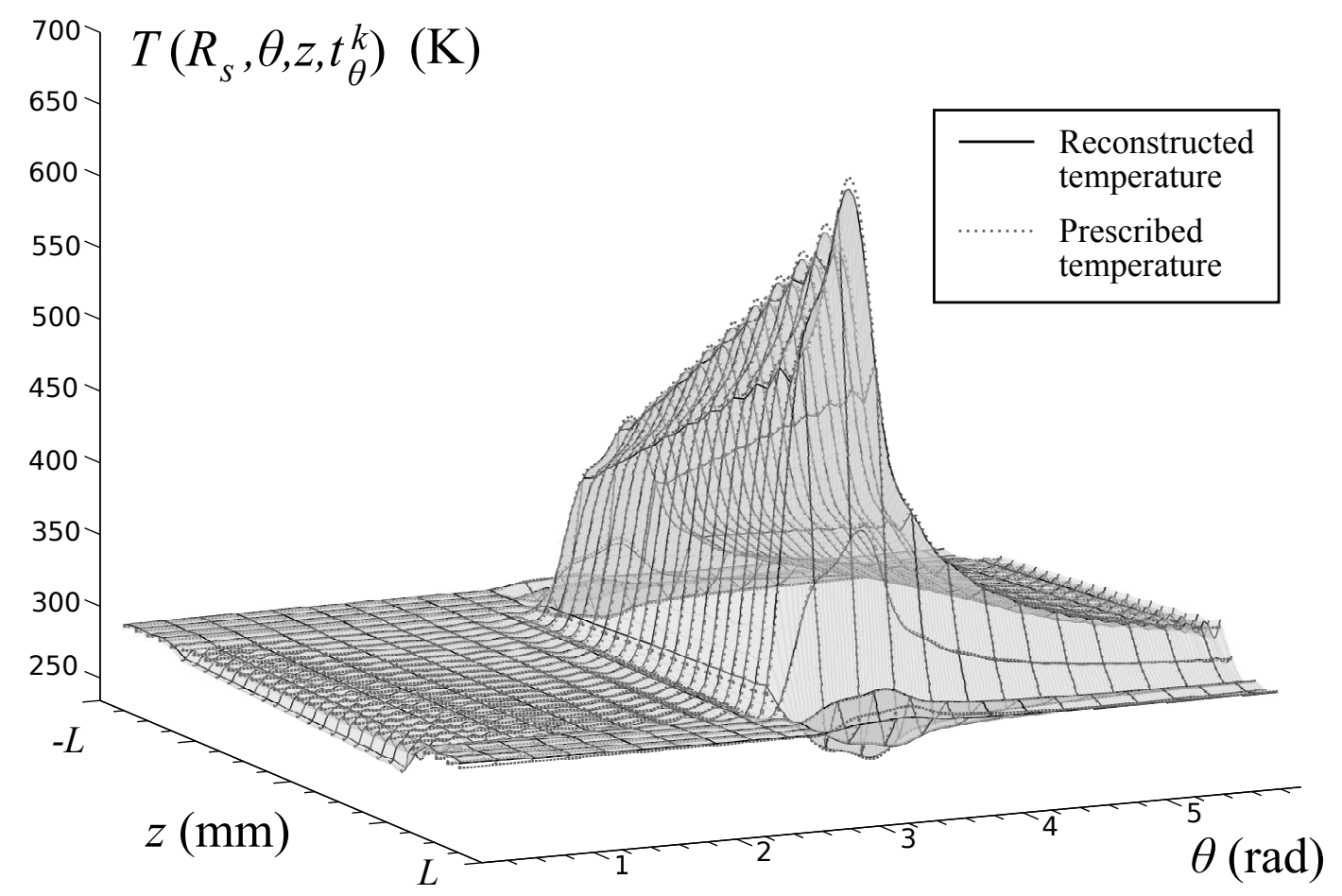

Figure 17: First cycle, reconstructed temperatures at the surface of the roll without noise for $N_{z}^{i}=1000$ and $N_{\theta}^{i}=1000$

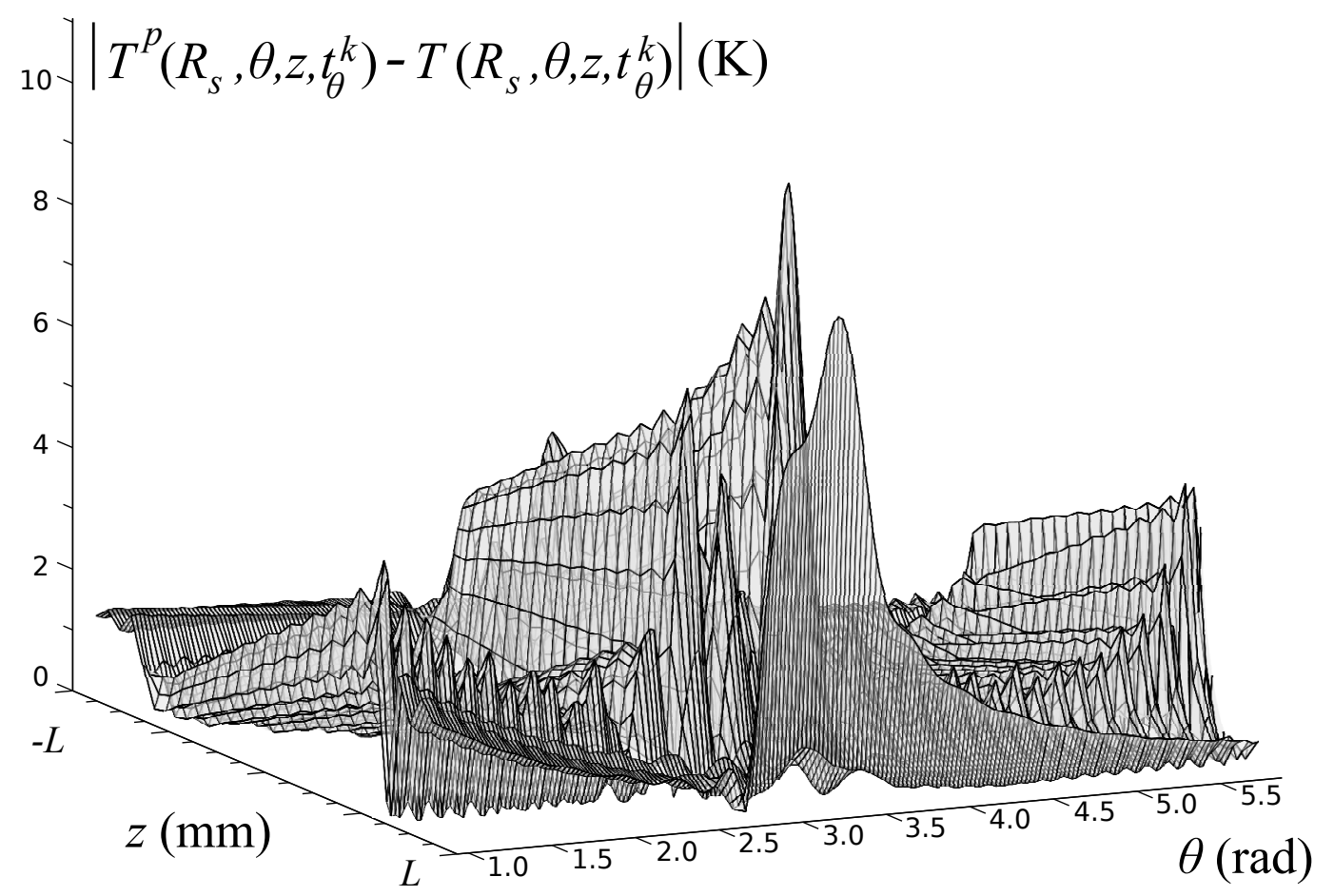

Figure 18: First cycle, error of reconstruction without noise for $N_{z}^{i}=1000$ and $N_{\theta}^{i}=1000$ 


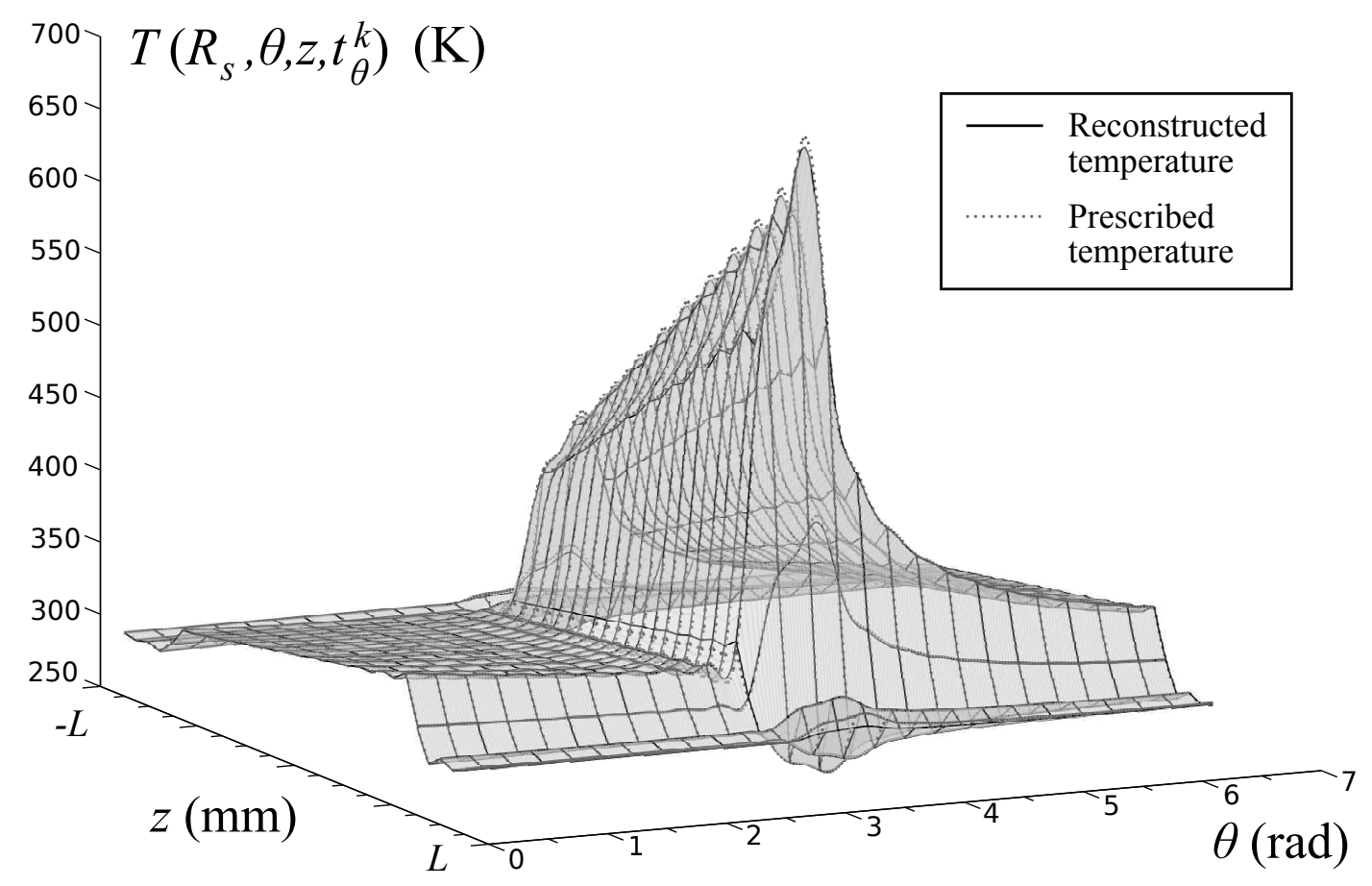

Figure 19: Cycle after $10 \mathrm{~min}$, reconstructed temperatures at the surface of the roll without noise for $N_{z}^{i}=1000$ and $N_{\theta}^{i}=1000$

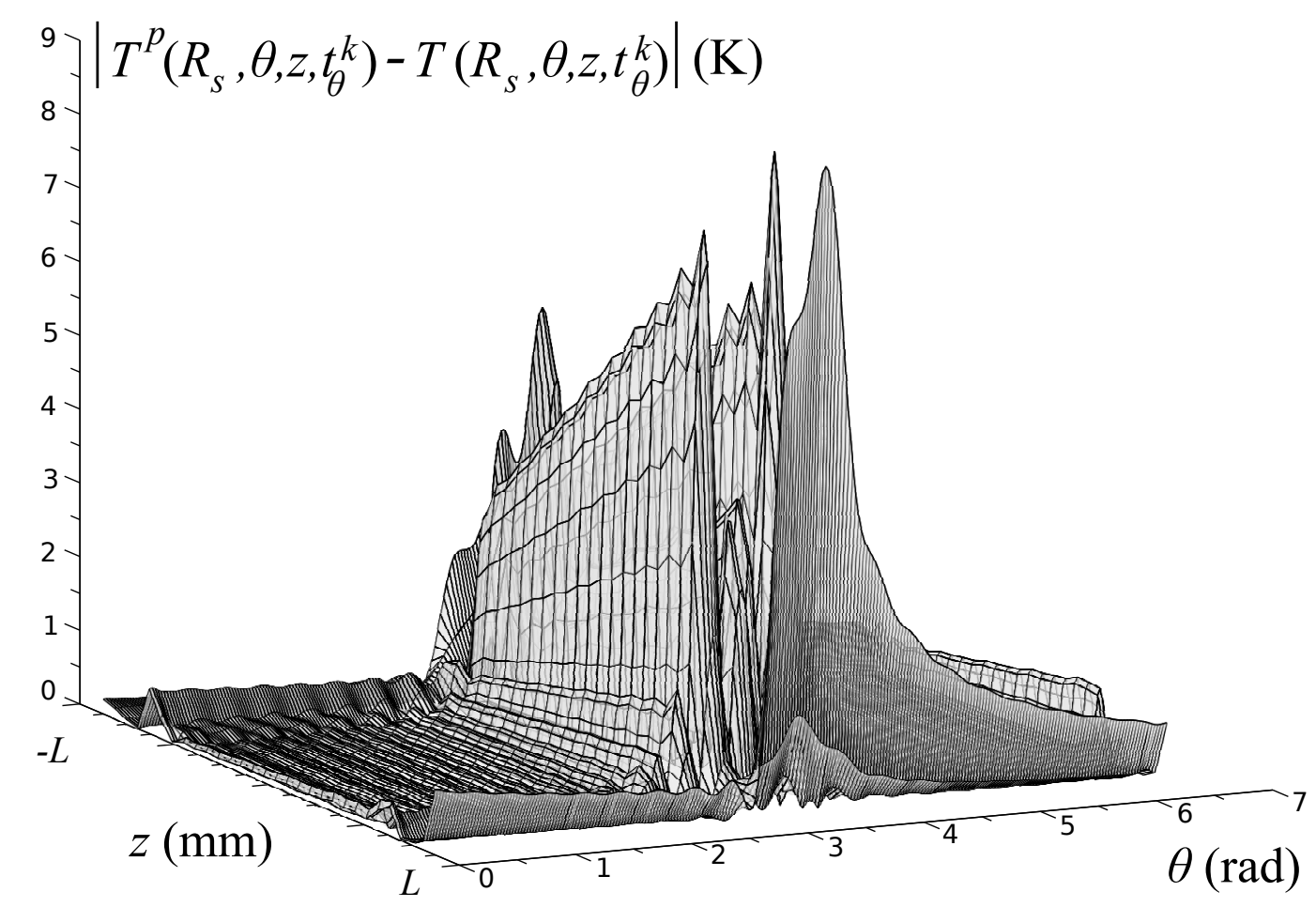

Figure 20: Cycle after $10 \mathrm{~min}$, error of reconstruction without noise for $N_{z}^{i}=1000$ and $N_{\theta}^{i}=1000$ 


\begin{tabular}{|c|cc|cc|c|}
\cline { 2 - 6 } \multicolumn{1}{c|}{} & \multicolumn{4}{c|}{$\epsilon$} & CPU time \\
\cline { 2 - 6 } \multicolumn{1}{c|}{} & Without noise & With noise & $(\mathrm{s})$ \\
\hline First cycle & $1.1 \%$ & Fig.13 & $1.8 \%$ & Fig.21 & $\simeq 0.07$ \\
\hline Cycle after 10 min & $1.0 \%$ & Fig.15 & $1.7 \%$ & Fig.22 & $\simeq 0.07$ \\
\hline
\end{tabular}

Table 3: Quantified error for $N_{z}^{i}=100$ and $N_{\theta}^{i}=1000$

\begin{tabular}{|c|rc|cc|c|}
\cline { 2 - 6 } \multicolumn{1}{c|}{} & \multicolumn{4}{c|}{$\epsilon$} & CPU time \\
\cline { 2 - 6 } \multicolumn{1}{c|}{} & Without noise & \multicolumn{2}{c|}{ With noise } & $(\mathrm{s})$ \\
\hline First cycle & $0.7 \%$ & Fig.17 & $1.6 \%$ & Fig.23 & $\simeq 0.5$ \\
\hline Cycle after 10 min & $0.35 \%$ & Fig.19 & $1.4 \%$ & Fig.24 & $\simeq 0.5$ \\
\hline
\end{tabular}

Table 4: Quantified error for $N_{z}^{i}=1000$ and $N_{\theta}^{i}=1000$

\subsection{Noise sensitivity}

The measurements are carried out practically with noise. Artificial noise (uniform law and amplitude $1 \mathrm{~K}$ ) is added to the inputs. Then the reconstruction is calculated like in section 8.3. The outputs of the method compared to the prescribed temperatures at the surface of the roll are given in Figures 21 and 23 for the first cycle and Figures 22 and 24 for the cycle after 10 minutes. The quantified results are listed in Table 3 . The reconstruction is satisfying and therefore the noise sensitivity does not compromise the method.

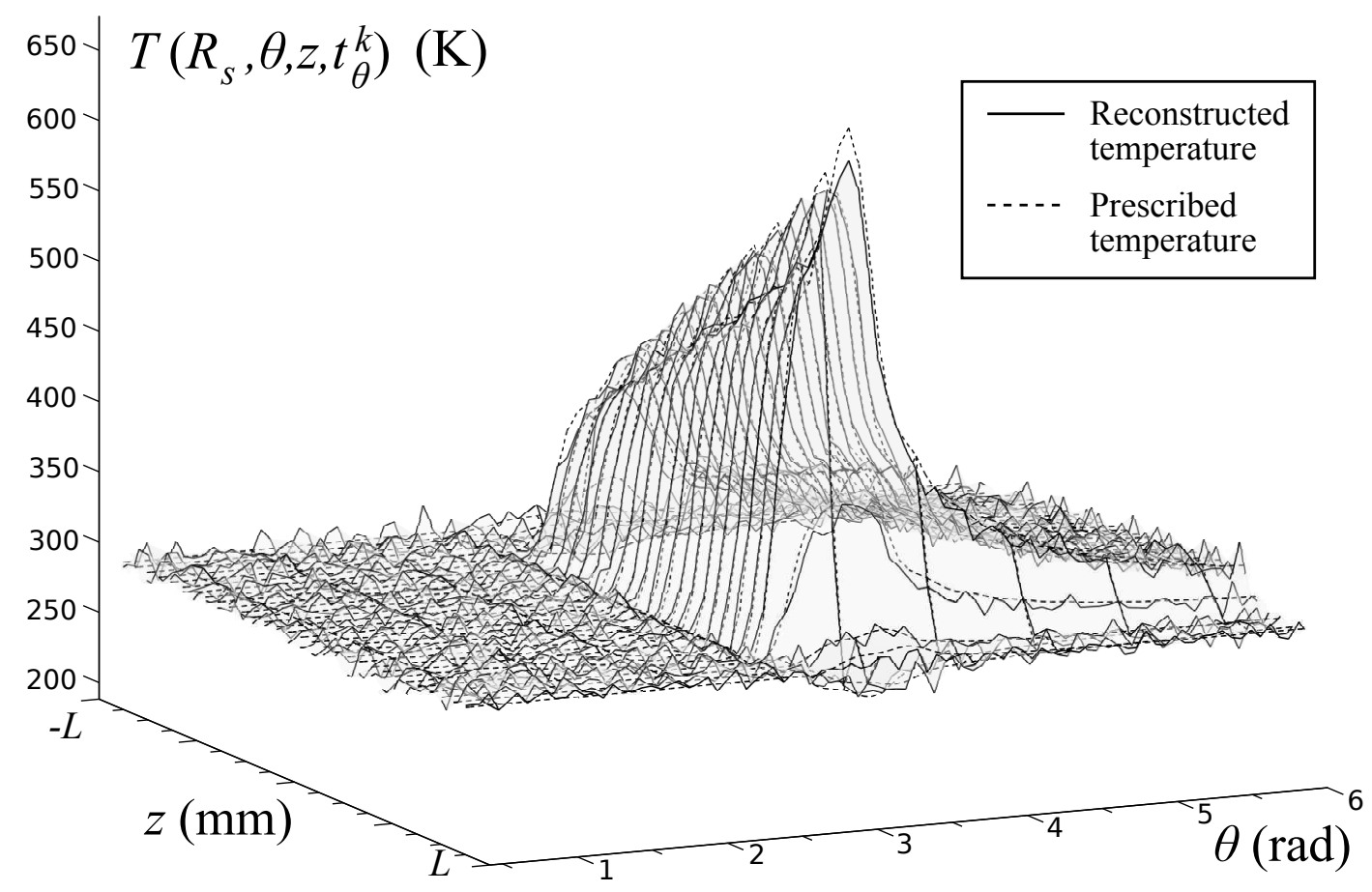

Figure 21: First cycle, reconstructed temperatures at the surface of the roll with noise for $N_{z}^{i}=100$ and $N_{\theta}^{i}=1000$ 


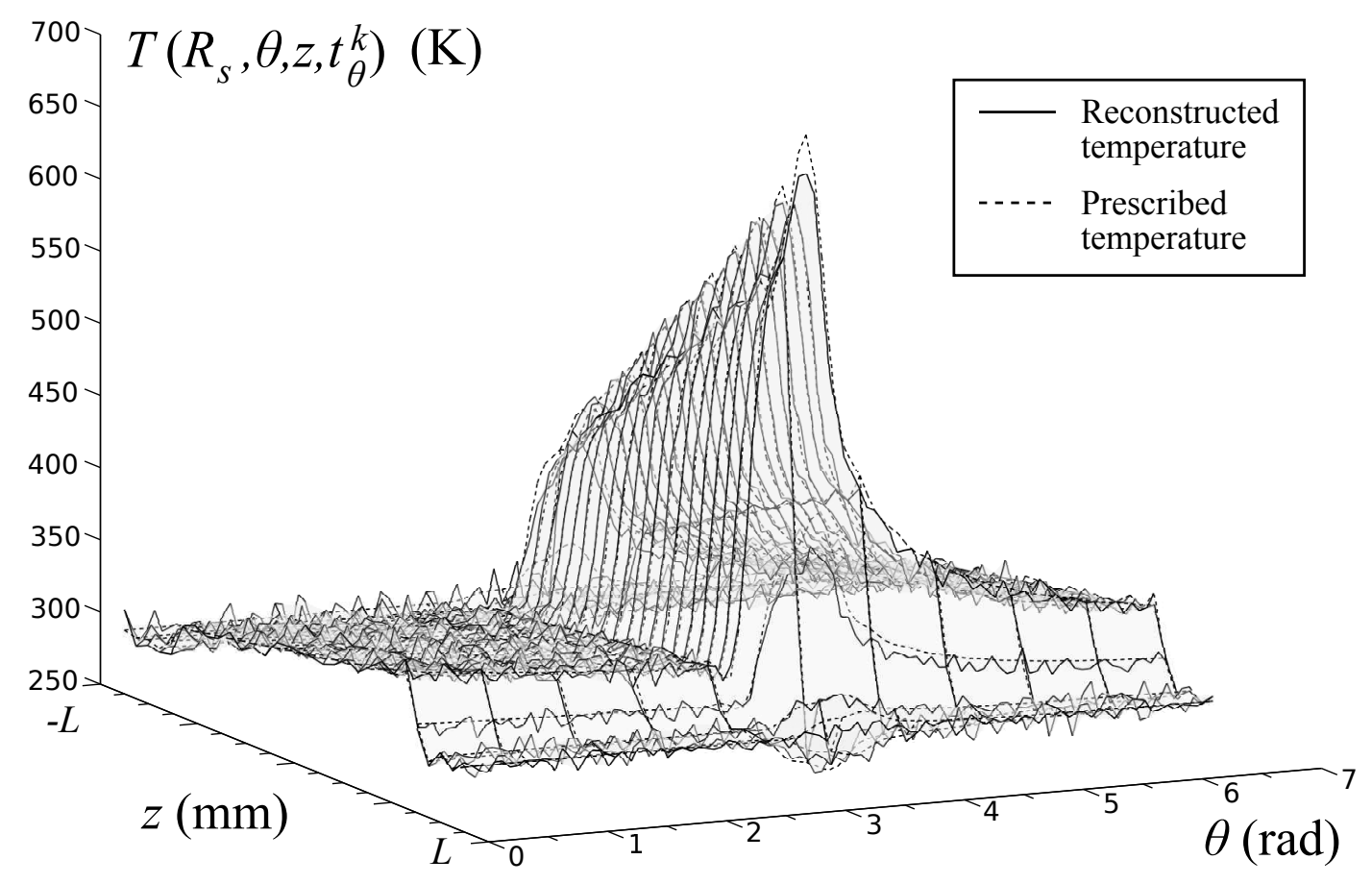

Figure 22: Cycle after $10 \mathrm{~min}$, reconstructed temperatures at the surface of the roll with noise for $N_{z}^{i}=100$ and $N_{\theta}^{i}=1000$

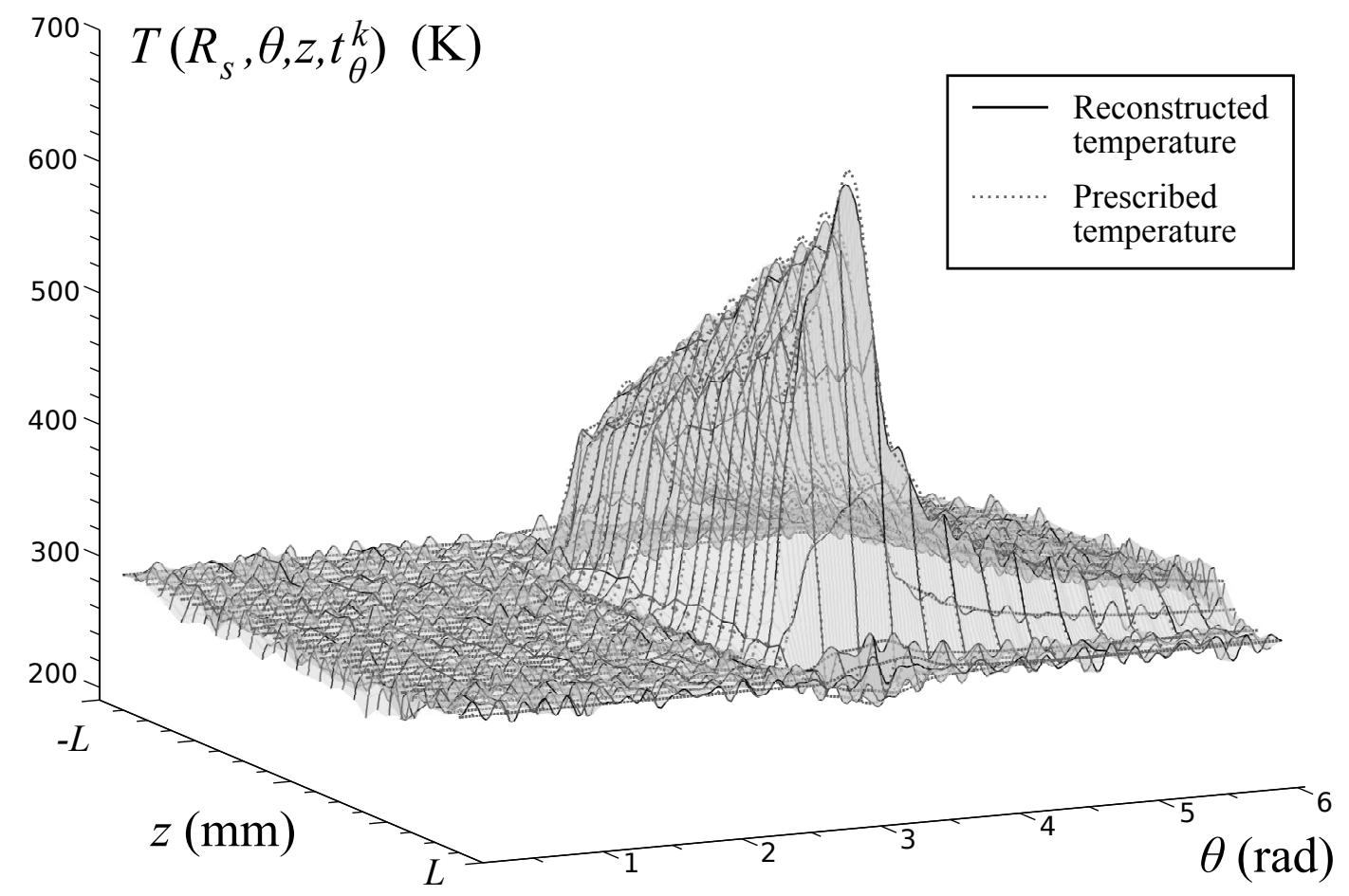

Figure 23: First cycle, reconstructed temperatures at the surface of the roll with noise for $N_{z}^{i}=1000$ and $N_{\theta}^{i}=1000$ 


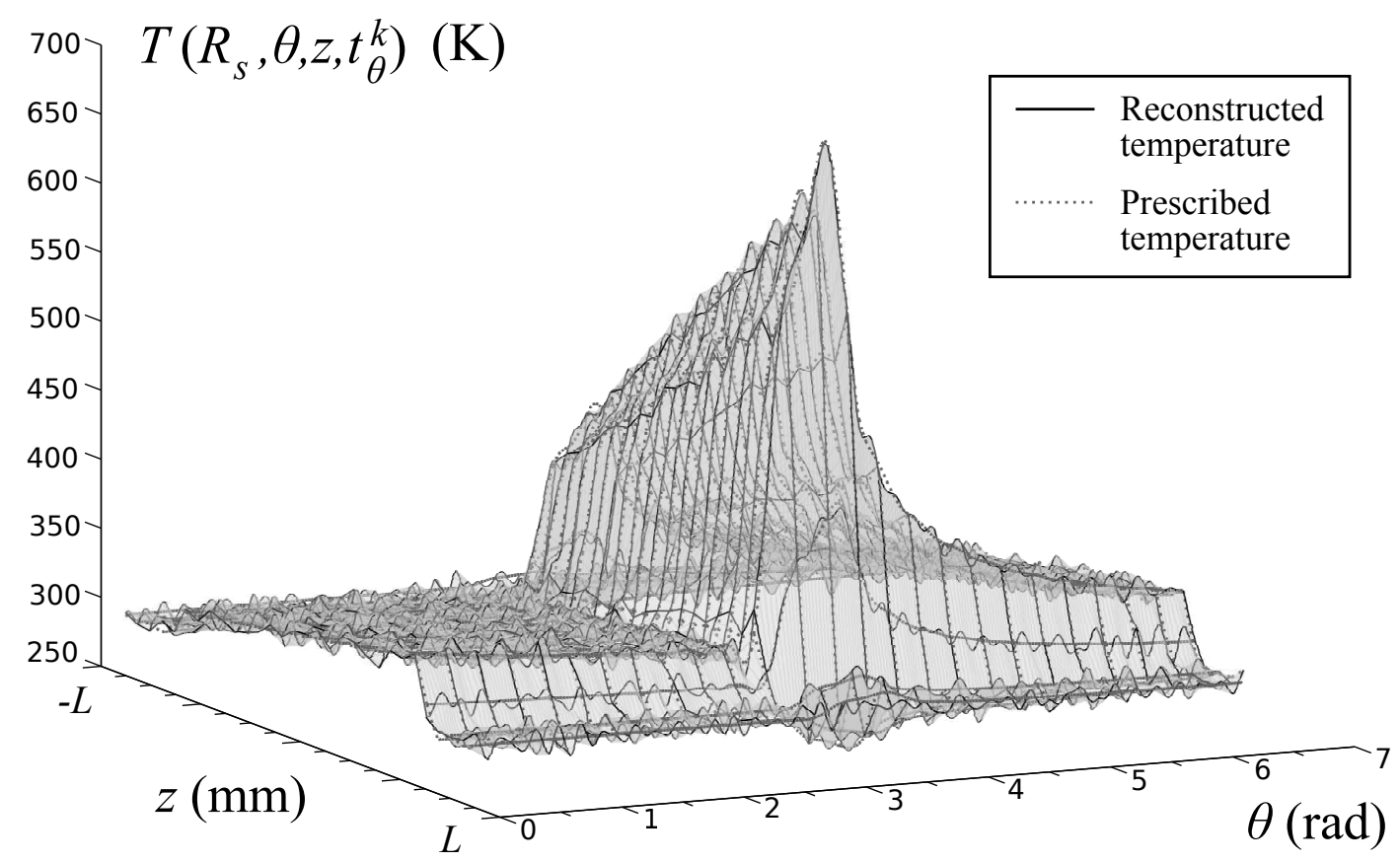

Figure 24: Cycle after $10 \mathrm{~min}$, reconstructed temperatures at the surface of the roll with noise for $N_{z}^{i}=1000$ and $N_{\theta}^{i}=1000$

\subsection{Sensor depth}

Technologically the temperature sensor depth is known with an error. Therefore it is a good result if the inverse method is not very sensitive to uncertainties about the sensor depth. Here a $10 \%$ error of the depth is considered for each thermocouple. Therefore, inputs with random errors (magnitude $e=0.05 \mathrm{~mm}$ ) done on $R_{m}$ are considered and reported in Figure 25(a). The reconstructed temperature for the first cycle is shown in Figures 25(b) and 25(c), and the quantified errors are listed in Table.4. Temperature reconstruction is not very sensitive to a $10 \%$ error of the temperature sensor depth.

\begin{tabular}{|c|cc|cc|c|}
\cline { 2 - 5 } \multicolumn{1}{c|}{} & \multicolumn{4}{c|}{$\epsilon$} & CPU time \\
\cline { 2 - 5 } \multicolumn{1}{c|}{} & Without noise & With noise & $(\mathrm{s})$ \\
\hline First cycle & $1.0 \%$ & Fig.25(b) & $2.0 \%$ & Fig.25(c) & $\simeq 0.5$ \\
\hline
\end{tabular}

Table 5: Quantified error considering 10\% error of the depth of each thermocouple, for $N_{z}^{i}=1000$ and $N_{\theta}^{i}=1000$ 


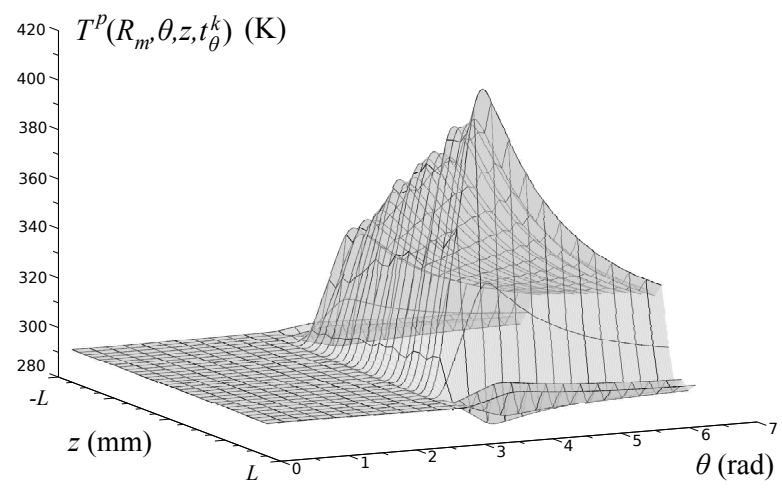

(a) Inputs with $10 \%$ error of the sensor depth

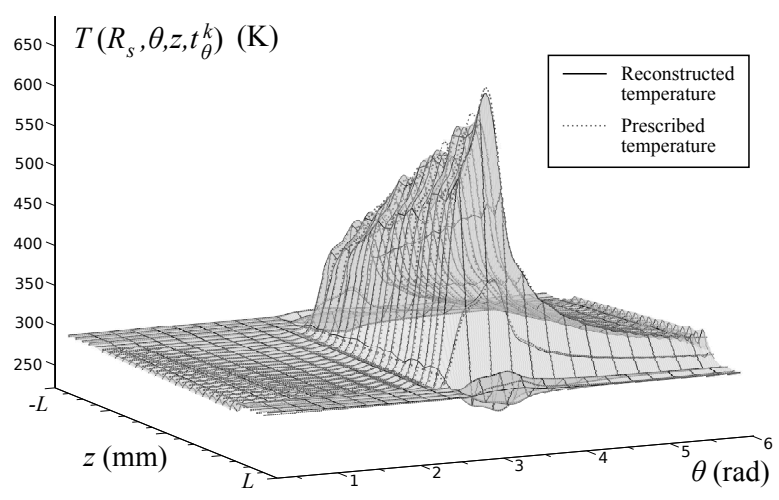

(b) Reconstruction without noise

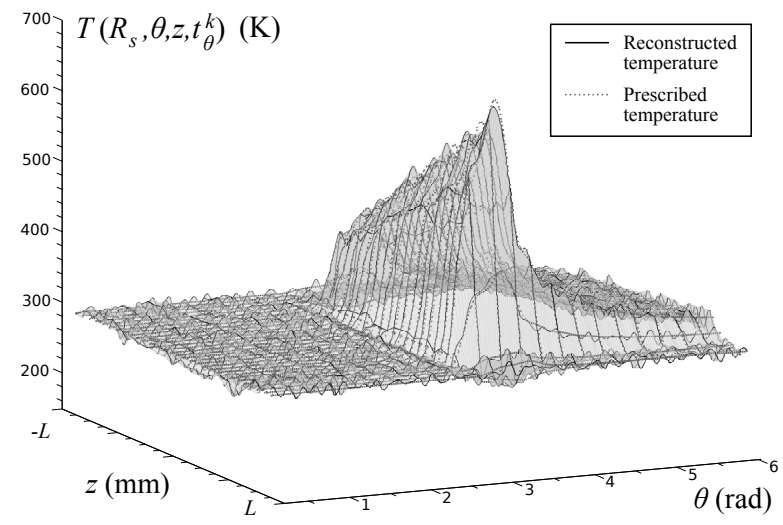

(c) Reconstruction with noise (amplitude 1K)

Figure 25: Sensor depth sensitivity

\section{Comparison with previous work}

As detailed in section 2, the present inverse solution improves a previous work [18] where three unnecessary assumptions were made. The interest of using a 3D inverse method compared with a 2D inverse solution has been highlighted. In this section, only the improvement of analytical solution is emphasized. In figure 26, reconstructions of prescribed temperatures for the first cycle are shown for both 2D previous work [18] and present 3D inverse method for two values of $z$. It can be noted a slightly better accuracy for the present work. The unnecessary assumptions of the previous work [18] led to an underestimation of temperature at the beginning of the cycle and at the exit of the roll gap. 


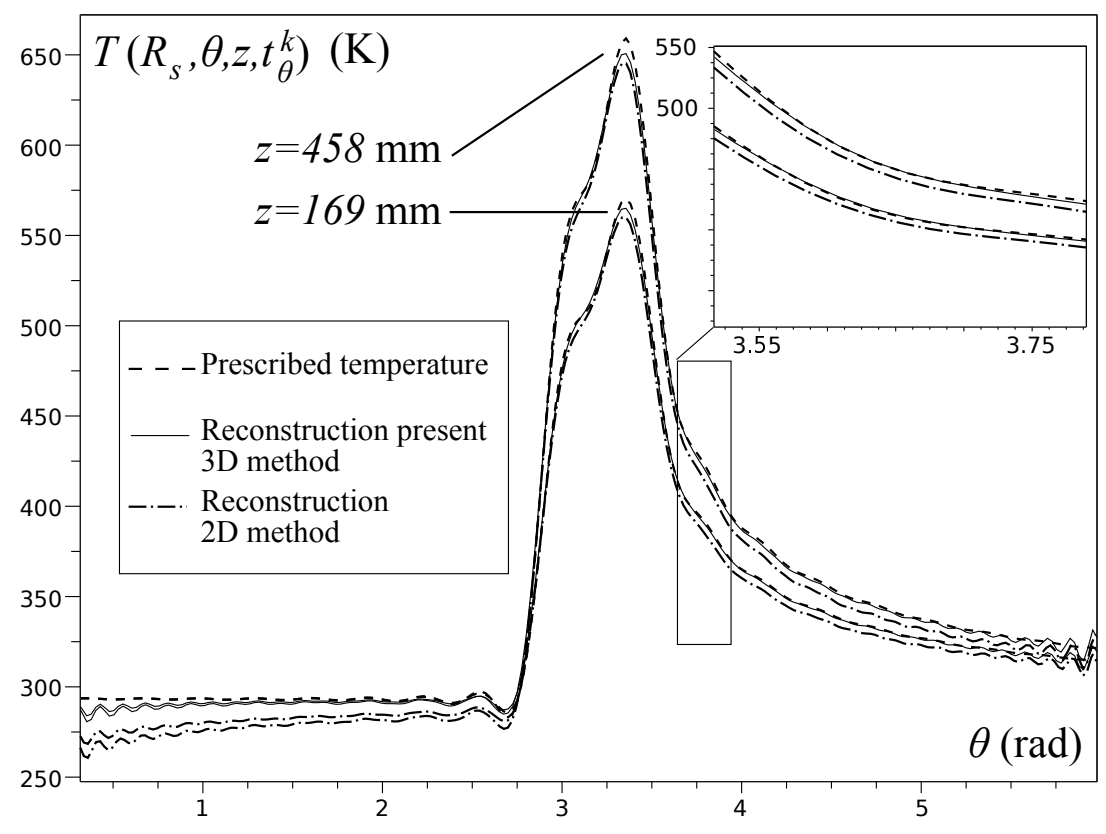

Figure 26: Comparison with previous work

\section{Computation time}

The computation time is studied to rapidly optimise the industrial parameters during the rolling process. The principle is to write the solution in matrix form or in hypermatrix form (considering the three levels of sum). The matrices (or hypermatrices) can be computed off-line (i.e., before the rolling process) and be stocked in a library (the frequency of acquisition being known). The solution (8) is written by using (16)+(21) in hypermatrice form. The only quantities to compute on-line are $A_{n, p}^{k}$ and $B_{n, p}^{k}$. Considering (14) it is convenient to use the Fast Fourier Transform (fft). A well known method to improve the quality of these integrals is to interpolate the discrete inputs with cubic spline and to apply the fft. Comparing with the two dimensional case (CPU time $0.05 \mathrm{sec}$ by cycle after Weisz-Patrault et al. [18]) these fft take more time because of the double integrals, however the number of points of interpolation has been reduced to get similar computation times $(0.07 \mathrm{sec}$ by cycle $)$ without comprising the integral quality. A more accurate computation can be obtained in 0.5 second by cycle. This computation time is promising for a real time computation and has been obtained for a Quadcore $2.8 \mathrm{GHz}$ processor and is the time displayed by Scilab 5.3. A significant improvement could be the implementation in a compiled code written for example in $\mathrm{C}++$.

\section{Conclusion}

A three dimensional inverse analytical method has been developed mainly to estimate the temperature distribution at the surface of the roll. Heat fluxes can be derived from the method also. The temperature (and heat flux) can be obtained in the whole roll. The method interprets the measurements of several thermocouples fully embedded under the surface of the roll and aligned along the axial direction. The numerical results presented in this paper are satisfying for an industrial rotation speed. The measurements are carried out practically with noise. Noise sensitivity has been studied by adding artificial random numbers to the inputs, and accuracy has not been compromised. Sensitivity to sensor depth errors has been studied and is not critical. This enables experimental estimations for flatness and crown control. The advantages of the present truly 3D solution compared with the previous work [18] have been highlighted. Moreover, the main advantage of this contribution, compared with the few existing 3D iterative methods, is the very short computation times: 0.07 second for each cycle for a rough computation or 0.5 second by cycle for a more accurate computation (CPU times are obtained for a quadcore $2.8 \mathrm{GHz}$ processor and is the time displayed by Scilab 5.3). Therefore the method is promising for a real time computation in order to optimise the flatness control devices and crown correction tools during industrial rolling processes. An industrial sensor can be developed on the basis of this contribution as a simple tool for on-line industrial monitoring and control of flatness and crown of the product. 


\section{Appendix A. Family of solutions}

The unsteady heat equation relative to an Eulerian reference, expressed in polar coordinates, where the thermal properties of the material are assumed to not depend on the temperature is given by the equation:

$$
\frac{\partial^{2} T}{\partial r^{2}}+\frac{1}{r} \frac{\partial T}{\partial r}+\frac{1}{r^{2}} \frac{\partial^{2} T}{\partial \theta^{2}}+\frac{\partial^{2} T}{\partial z^{2}}=\frac{1}{D}\left(\frac{\partial T}{\partial t}+\omega \frac{\partial T}{\partial \theta}\right)
$$

The solution ( $2 \pi$-periodic for $\theta$ at any time) can be developed in Fourier series:

$$
T(r, \theta, z, t)=\sum_{n=-\infty}^{+\infty} F_{n}(r, z, t) \exp (\operatorname{in} \theta)
$$

The $n$th Fourier coefficients is sought such as:

$$
F_{n}(r, z, t)=a_{n}(r) b_{n}(z) c_{n}(t)
$$

Thus:

$$
b_{n}(z) c_{n}(t)\left(a_{n}^{\prime \prime}(r)+\frac{1}{r} a_{n}^{\prime}(r)-\frac{n^{2}}{r^{2}} a_{n}(r)\right)+a_{n}(r) b_{n}^{\prime \prime}(z) c_{n}(t)=\frac{a_{n}(r) b_{n}(z)}{D}\left(c_{n}^{\prime}(t)+i \omega n c_{n}(t)\right)
$$

The condition $T(r, \theta, t)>0$ is verified ( $T$ expressed in Kelvin) therefore:

$$
\frac{1}{a_{n}(r)}\left(a_{n}^{\prime \prime}(r)+\frac{1}{r} a_{n}^{\prime}(r)-\frac{n^{2}}{r^{2}} a_{n}(r)\right)+\frac{b_{n}^{\prime \prime}(z)}{b_{n}(z)}=\frac{1}{D c_{n}(t)}\left(c_{n}^{\prime}(t)+i \omega n c_{n}(t)\right)
$$

Both terms are functions of independent variables, thus two complex numbers $C_{1}$ and $C_{2}$ exist such as:

$$
\left\{\begin{array}{l}
\frac{1}{a_{n}(r)}\left(a_{n}^{\prime \prime}(r)+\frac{1}{r} a_{n}^{\prime}(r)-\frac{n^{2}}{r^{2}} a_{n}(r)\right)=C_{1}-\frac{b_{n}^{\prime \prime}(z)}{b_{n}(z)}=C_{2} \\
\frac{1}{D c_{n}(t)}\left(c_{n}^{\prime}(t)+i \omega n c_{n}(t)\right)=C_{1}
\end{array}\right.
$$

Thus:

$$
\left\{\begin{array}{l}
a_{n}(r)=J_{n}\left(\sqrt{-C_{2}} r\right) \\
b_{n}(z)=\exp \left(\sqrt{C_{1}-C_{2}} z\right) \\
c_{n}(t)=\exp \left(\left(D C_{1}-i \omega n\right) t\right)
\end{array}\right.
$$

where $J_{n}$ is the Bessel function of the first kind of the order $n$.

By introducing $\tau=-1 /\left(D C_{1}-i \omega n\right)$ and $\alpha=\sqrt{C_{1}-C_{2}}$ it is obtained that the following function is a solution of (A.1).

$$
\gamma J_{n}\left(\sqrt{\frac{1}{D \tau}-\frac{i \omega n}{D}+\alpha^{2}} r\right) \exp \left(-\frac{t}{\tau}\right) \exp (\operatorname{in} \theta) \exp (\alpha z)
$$

\section{Appendix B. Direct analytical solution}

An analytical solution is sought for the problem of a roll heated by a surrounding temperature which creates a heat flux equal to the difference between the surrounding temperature and the temperature at the surface of the roll multiplied by a heat transfer coefficient. Here HTC is taken constant which is discussed in Section 8 . The surrounding temperature (called $\left.T^{*}\right)$ is everywhere $T_{a}$ but in a part $(\theta, z) \in[\pi-\Theta, \pi+\Theta] \times[-L+Z, L-Z]$ where it is slope as defined in Figure B.27. 


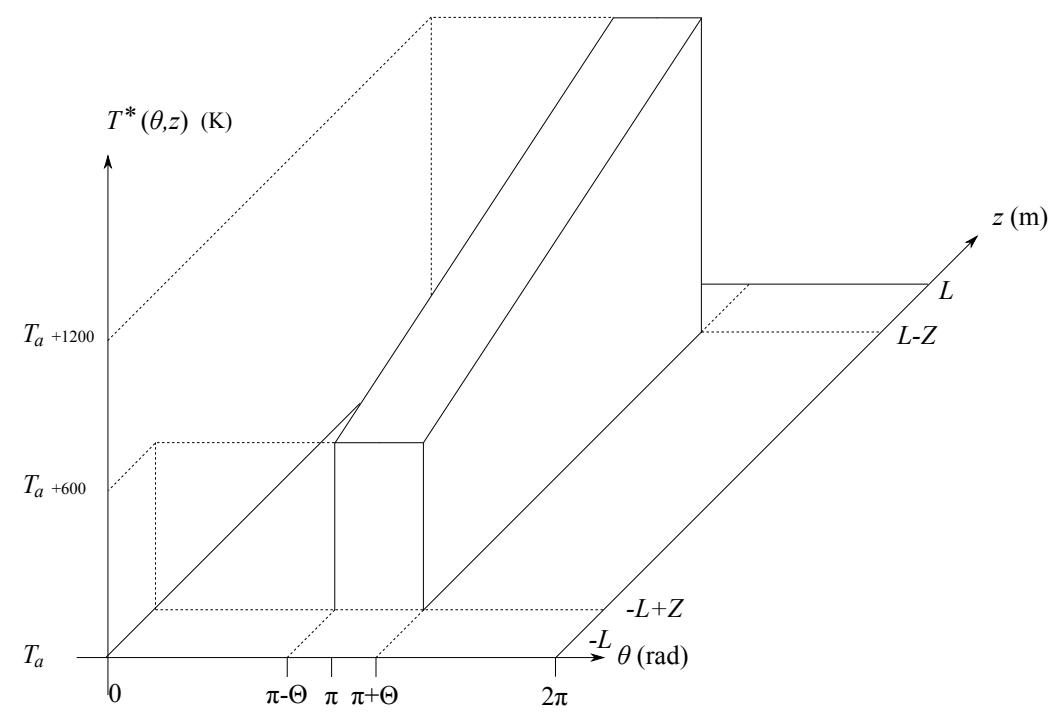

Figure B.27: Surrounding temperature: $T^{*}$

Therefore an expansion into a Fourier series gives:

$$
T^{*}(\theta, z)=\sum_{n=-N_{2}}^{N_{2}} \sum_{p=0}^{P_{2}}\left[a_{n, p}^{*} \cos \left(\frac{\pi p z}{L}\right)+b_{n, p}^{*} \sin \left(\frac{\pi(2 p+1) z}{2 L}\right)\right] \exp (\operatorname{in} \theta)
$$

The coefficients $a_{n, p}^{*}$ and $b_{n, p}^{*}$ are defined as follows:

$$
\begin{aligned}
& \forall n \in \mathbb{Z}, \forall p \geq 1\left\{\begin{array}{l}
a_{n, p}^{*}=\frac{1}{2 L \pi} \int_{-L}^{L} \int_{0}^{2 \pi} T^{*}(\theta, z) \exp (-i n \theta) \cos \left(\frac{p \pi z}{L}\right) \mathrm{d} \theta \mathrm{d} z \\
b_{n, p}^{*}=\frac{1}{2 L \pi} \int_{-L}^{L} \int_{0}^{2 \pi} T^{*}(\theta, z) \exp (-i n \theta) \sin \left(\frac{(2 p+1) \pi z}{2 L}\right) \mathrm{d} \theta \mathrm{d} z
\end{array}\right. \\
& \forall n \in \mathbb{Z} \\
& \left\{\begin{array}{l}
a_{n, 0}^{*}=\frac{1}{4 L \pi} \int_{-L}^{L} \int_{0}^{2 \pi} T^{*}(\theta, z) \exp (-i n \theta) \mathrm{d} \theta \mathrm{d} z \\
b_{n, 0}^{*}=\frac{1}{2 L \pi} \int_{-L}^{L} \int_{0}^{2 \pi} T^{*}(\theta, z) \exp (-i n \theta) \sin \left(\frac{\pi z}{2 L}\right) \mathrm{d} \theta \mathrm{d} z
\end{array}\right.
\end{aligned}
$$

For the purpose of validating the method only a few terms are considered (i.e., $N_{2}=20$ and $P_{2}=14$ ). Therefore the real profile of $T^{*}$ is given in Figure B.28 


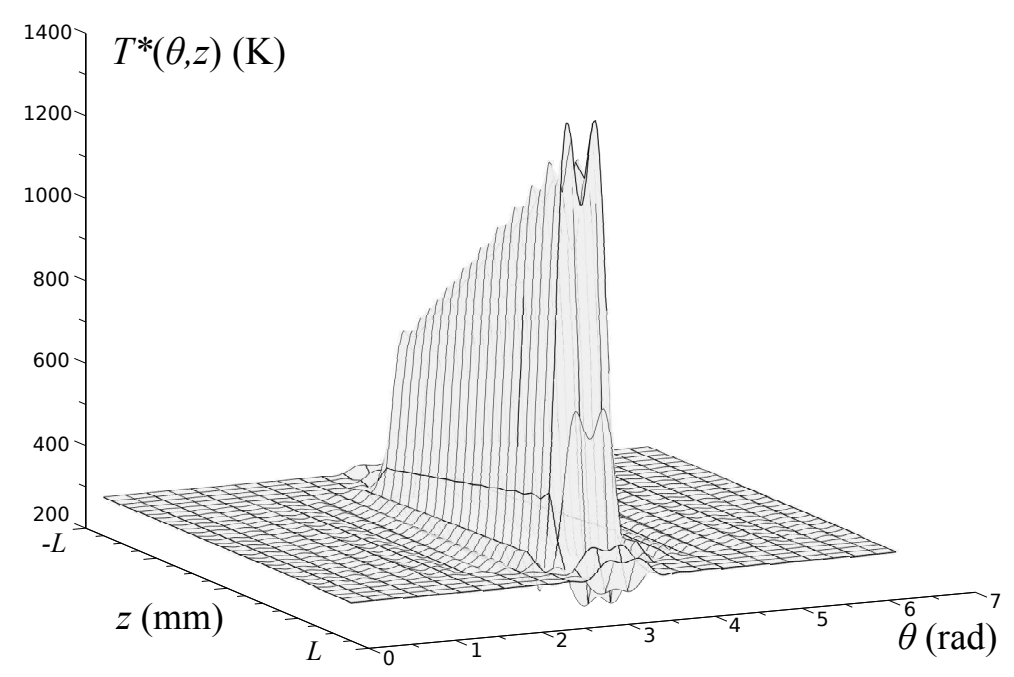

Figure B.28: Surrounding temperature: $T^{*}$

The solution of the problem is sought in the form of (7):

$$
\begin{array}{r}
T^{p}(r, \theta, z, t)=\sum_{n=-N_{2}}^{N_{2}} \sum_{p=0}^{P_{2}}\left[a_{n, p} J_{n}\left(\zeta_{n, p} r\right) \cos \left(\frac{\pi p z}{L}\right)+b_{n, p} J_{n}\left(\chi_{n, p} r\right) \sin \left(\frac{\pi(2 p+1) z}{2 L}\right)\right] \exp (\operatorname{in} \theta) \\
+\sum_{n=-N_{2}}^{N_{2}} \sum_{p=0}^{P_{2}} \sum_{q=1}^{Q_{2}} c_{n, p, q} J_{n}\left(r \sqrt{\frac{1}{D \tau_{n, p, q}^{*}}-\frac{i \omega n}{D}-\left(\frac{\pi p}{L}\right)^{2}}\right) \exp \left(\frac{-t}{\tau_{n, p, q}^{*}}\right) \exp (\operatorname{in} \theta) \cos \left(\frac{\pi p z}{L}\right) \\
+\sum_{n=-N_{2}}^{N_{2}} \sum_{p=0}^{P_{2}} \sum_{q=1}^{Q_{2}} d_{n, p, q} J_{n}\left(r \sqrt{\frac{1}{D v_{n, p, q}^{*}}-\frac{i \omega n}{D}-\left(\frac{\pi(2 p+1)}{2 L}\right)^{2}}\right) \exp \left(\frac{-t}{v_{n, p, q}^{*}}\right) \exp (\operatorname{in} \theta) \sin \left(\frac{\pi(2 p+1) z}{2 L}\right)
\end{array}
$$

where $a_{n, p}, b_{n, p}, c_{n, p, q}$ and $d_{n, p, q}$ are complex numbers and $N_{2}, P_{2}$ and $Q_{2}$ are integers.

The boundary condition is:

$$
\lambda \frac{\partial T^{p}}{\partial r}\left(R_{s}, \theta, z, t\right)=\mathrm{HTC} \times\left(T^{*}(\theta, z)-T^{p}\left(R_{s}, \theta, z, t\right)\right)
$$

It is convenient to introduce:

$$
y_{n, q}=R_{s} \sqrt{\frac{1}{D \tau_{n, p, q}^{*}}-\frac{i \omega n}{D}-\left(\frac{\pi p}{L}\right)^{2}}=R_{s} \sqrt{\frac{1}{D v_{n, p, q}^{*}}-\frac{i \omega n}{D}-\left(\frac{\pi(2 p+1)}{2 L}\right)^{2}}
$$

It can be noted that the notation $y_{n, q}$ is abusive because the definition involves clearly the index $p$. However, the boundary condition (B.4) gives:

$$
\frac{\lambda y_{n, q}}{R_{s}} J_{n}^{\prime}\left(y_{n, q}\right)+\mathrm{HTC} \times J_{n}\left(y_{n, q}\right)=0
$$

Therefore $y_{n, q}$ do not depend on $p$ and are calculated numerically by taking the successive positive zeros of the functions:

$$
y \mapsto h_{n}(y)=\lambda \frac{y}{R_{s}} J_{n}^{\prime}(y)+\mathrm{HTC} \times J_{n}(y)
$$

Figure B.29 shows an example. 


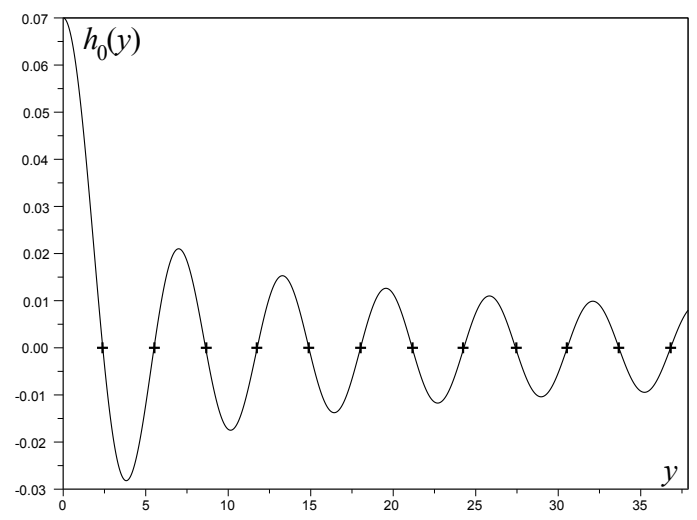

Figure B.29: Successive zeros of $h_{0}(x)$

Therefore:

$$
\left\{\begin{array}{l}
\tau_{n, p, q}^{*}=\frac{1}{D}\left(\left(\frac{y_{n, q}}{R_{S}}\right)^{2}+\frac{i \omega n}{D}+\left(\frac{\pi p}{L}\right)^{2}\right)^{-1} \\
v_{n, p, q}^{*}=\frac{1}{D}\left(\left(\frac{y_{n, q}}{R_{s}}\right)^{2}+\frac{i \omega n}{D}+\left(\frac{\pi(2 p+1)}{2 L}\right)^{2}\right)^{-1}
\end{array}\right.
$$

The boundary condition (B.4) gives also for terms independent on time:

$$
\left\{\begin{array}{l}
a_{0,0}=a_{0,0}^{*} \\
a_{n, p}=\frac{\mathrm{HTC} \times a_{n, p}^{*}}{\lambda \zeta_{n, p} J_{n}^{\prime}\left(\zeta_{n, p} R_{s}\right)+\mathrm{HTC} \times J_{n}\left(\zeta_{n, p} R_{s}\right)} \\
b_{0,0}=\frac{\mathrm{HTC} \times b_{0,0}^{*}}{\lambda \chi_{0,0} J_{0}^{\prime}\left(\chi_{0,0} R_{s}\right)+\mathrm{HTC} \times J_{0}\left(\chi_{0,0} R_{s}\right)} \\
b_{n, p}=\frac{\mathrm{HTC} \times b_{n, p}^{*}}{\lambda \chi_{n, p} J_{n}^{\prime}\left(\chi_{n, p} R_{s}\right)+\mathrm{HTC} \times J_{n}\left(\chi_{n, p} R_{s}\right)}
\end{array}\right.
$$

The initial condition is:

$$
T^{p}(r, \theta, z, 0)=T_{a}
$$

The initial condition (B.10) gives:

$$
\left\{\begin{array}{l}
\sum_{q=1}^{Q_{2}} c_{0,0, q} J_{0}\left(\frac{y_{0, q} r}{R_{s}}\right)=T_{a}-a_{0,0}=u_{0,0}^{*}(r) \\
\sum_{q=1}^{Q_{2}} c_{n, p, q} J_{n}\left(\frac{y_{n, q} r}{R_{s}}\right)=-a_{n, p} J_{n}\left(\zeta_{n, p} r\right)=u_{n, p}^{*}(r) \\
\sum_{q=1}^{Q_{2}} d_{0,0, q} J_{0}\left(\frac{y_{0, q} r}{R_{s}}\right)=-b_{0,0}=v_{0,0}^{*}(r) \\
\sum_{q=1}^{Q_{2}} d_{n, p, q} J_{n}\left(\frac{y_{n, q} r}{R_{s}}\right)=-b_{n, p} J_{n}\left(\chi_{n, p} r\right)=v_{n, p}^{*}(r)
\end{array}\right.
$$

In their previous work Weisz-Patrault et al. [18] solved this kind of equation by descritizing, but there exists an analytical solution which is used in the present paper. (B.11) is called a Dini expansion and is used by Sneddon 
[29, p.40] and demonstrated by Watson [30, p.596].

$$
\left\{\begin{array}{l}
c_{n, p, q}=\frac{2 y_{n, q}^{2}}{\left(y_{n, q}^{2}-n^{2}+\frac{R_{s}^{2} \mathrm{HTC}^{2}}{\lambda^{2}}\right) R_{s}^{2} J_{n}^{2}\left(y_{n, q}\right)} \int_{0}^{R_{s}} \rho u_{n, p}^{*}(\rho) J_{n}\left(y_{n, q} \frac{\rho}{R_{s}}\right) \mathrm{d} \rho \\
d_{n, p, q}=\frac{2 y_{n, q}^{2}}{\left(y_{n, q}^{2}-n^{2}+\frac{R_{s}^{2} \mathrm{HTC}^{2}}{\lambda^{2}}\right) R_{s}^{2} J_{n}^{2}\left(y_{n, q}\right)} \int_{0}^{R_{s}} \rho v_{n, p}^{*}(\rho) J_{n}\left(y_{n, q} \frac{\rho}{R_{s}}\right) \mathrm{d} \rho
\end{array}\right.
$$

By using (27) and (28) the expression of $c_{n, p, q}$ and $d_{n, p, q}$ reduce to (B.13).

$$
\left\{\begin{array}{l}
c_{0,0, q}=\frac{2 y_{0, q}\left(T_{a}-a_{0,0}\right) J_{1}\left(y_{0, q}\right)}{\left(y_{0, q}^{2}+\frac{R_{s}^{2} \mathrm{HTC}^{2}}{\lambda^{2}}\right) J_{0}^{2}\left(y_{0, q}\right)} \\
c_{n, p, q}=\frac{2 y_{n, q}^{2} a_{n, p}}{\left(y_{n, q}^{2}-n^{2}+\frac{R_{s}^{2} \mathrm{HTC}^{2}}{\lambda^{2}}\right) J_{n}^{2}\left(y_{n, q}\right)} \frac{\zeta_{n, p} R_{s} J_{n+1}\left(\zeta_{n, p} R_{s}\right) J_{n}\left(y_{n, q}\right)-y_{n, q} J_{n+1}\left(y_{n, q}\right) J_{n}\left(\zeta_{n, p} R_{s}\right)}{y_{n, q}^{2}-\zeta_{n, p}^{2} R_{s}^{2}} \\
d_{0,0, q}=\frac{2 y_{0, q}^{2} b_{0,0}}{\left(y_{0, q}^{2}+\frac{R_{s}^{2} \mathrm{HTC}^{2}}{\lambda^{2}}\right) J_{0}^{2}\left(y_{0, q}\right)} \frac{\chi_{0,0} R_{s} J_{1}\left(\chi_{0,0} R_{s}\right) J_{0}\left(y_{0, q}\right)-y_{0, q} J_{1}\left(y_{0, q}\right) J_{0}\left(\chi_{0,0} R_{s}\right)}{y_{0, q}^{2}-\chi_{0,0}^{2} R_{s}^{2}} \\
d_{n, p, q}=\frac{2 y_{n, q}^{2} b_{n, p}}{\left(y_{n, q}^{2}-n^{2}+\frac{R_{s}^{2} \mathrm{HTC}^{2}}{\lambda^{2}}\right) J_{n}^{2}\left(y_{n, q}\right)} \frac{\chi_{n, p} R_{s} J_{n+1}\left(\chi_{n, p} R_{s}\right) J_{n}\left(y_{n, q}\right)-y_{n, q} J_{n+1}\left(y_{n, q}\right) J_{n}\left(\chi_{n, p} R_{s}\right)}{y_{n, q}^{2}-\chi_{n, p}^{2} R_{s}^{2}}
\end{array}\right.
$$

\section{Appendix C. Fast Fourier Transform Algorithm}

The inverse method relies on the fast computation of following quantities ( $\delta_{p, 0}$ is the Kronecker symbol):

$$
\forall n \in \mathbb{Z}, \forall p \geq 0\left\{\begin{array}{l}
A_{n, p}^{k}=\frac{1}{\left(2+2 \delta_{p, 0}\right) L \pi} \int_{0}^{2 \pi}\left(\int_{-L}^{L} T^{m}(\theta, z, k) \cos \left(\frac{p \pi z}{L}\right) \mathrm{d} z\right) \exp (-i n \theta) \mathrm{d} \theta \\
B_{n, p}^{k}=\frac{1}{2 L \pi} \int_{0}^{2 \pi}\left(\int_{-L}^{L} T^{m}(\theta, z, k) \sin \left(\frac{(2 p+1) \pi z}{2 L}\right) \mathrm{d} z\right) \exp (-i n \theta) \mathrm{d} \theta
\end{array}\right.
$$

The fft algorithm of a signal $F\left(x_{l}\right)$ given for $l \in\left\{1 ; \ldots ; N_{F}\right\}$ computes the following vector $\left(p \in\left\{1 ; \ldots ; N_{F}\right\}\right)$ :

$$
\mathrm{fft}\left[F\left(x_{l}\right)\right]_{p}=\sum_{l=1}^{N_{F}} F\left(x_{l}\right) \exp \left(-i \frac{2 \pi(l-1)}{N_{F}} p\right)
$$

Thus, if the measured temperatures $T^{m}(\theta, z, k)$ are interpolated with cubic splines at $\theta_{j}=\left(2 \pi / N_{\theta}^{i}\right)(j-1)$ and $z_{l}=\left(2 L / N_{z}^{i}\right)(l-1)-L$, where the number of interpolation points are $N_{\theta}^{i}$ and $N_{z}^{i}$, then the Riemann approximation gives:

$$
\begin{aligned}
\int_{-L}^{L} T^{m}(\theta, z, k) \cos \left(\frac{p \pi z}{L}\right) \mathrm{d} z & \simeq \operatorname{Re}\left[\frac{2 L}{N_{z}^{i}} \sum_{l=1}^{N_{z}^{i}}(-1)^{p} T^{m}\left(\theta, z_{l}, k\right) \exp \left(-i \frac{2 \pi(l-1)}{N_{z}^{i}} p\right)\right] \\
& =\frac{2 L}{N_{z}^{i}} \operatorname{Re}\left[\mathrm{fft}\left[(-1)^{p} T^{m}\right]_{p}\right]
\end{aligned}
$$

Furthermore:

$$
\begin{aligned}
\int_{0}^{2 \pi} \operatorname{Re}\left[\mathrm{fft}\left[(-1)^{p} T^{m}\right]_{p}(\theta)\right] \exp (-i n \theta) \mathrm{d} \theta & \simeq \frac{2 \pi}{N_{\theta}^{i}} \sum_{j=1}^{N_{\theta}^{i}} \operatorname{Re}\left[\mathrm{fft}\left[(-1)^{p} T^{m}\right]_{p}\left(\theta_{j}\right)\right] \exp \left(-i \frac{2 \pi(j-1)}{N_{\theta}^{i}} n\right) \\
& =\frac{2 \pi}{N_{\theta}^{i}} \mathrm{ft}\left[\operatorname{Re}\left[\mathrm{ftt}\left[(-1)^{p} T^{m}\right]_{p}\right]\right]_{n}
\end{aligned}
$$


Finally:

$$
A_{n, p}^{k}=\frac{1}{\left(1+1 \delta_{p, 0}\right)} \frac{2}{N_{\theta}^{i} N_{z}^{i}} \mathrm{fft}\left[\operatorname{Re}\left[\mathrm{fft}\left[(-1)^{p} T^{m}\right]_{p}\right]\right]_{n}
$$

Moreover, since $\forall z \in[L, 3 L]$, $\sin ((2 p+1) \pi z /(2 L))=\sin ((2 p+1) \pi(2 L-z) /(2 L))$ :

$$
\int_{-L}^{L} T^{m}(\theta, z, k) \sin \left(\frac{(2 p+1) \pi z}{2 L}\right) \mathrm{d} z=\frac{1}{2} \int_{-L}^{3 L} \widetilde{T}^{m}(\theta, z, k) \sin \left(\frac{(2 p+1) \pi z}{2 L}\right) \mathrm{d} z
$$

where:

$$
\widetilde{T}^{m}(\theta, z, k)= \begin{cases}T^{m}(\theta, z, k) & z \in[-L, L] \\ T^{m}(\theta, 2 L-z, k) & z \in[L, 3 L]\end{cases}
$$

Thus, the interval $[-L, 3 L]$ is divided in $z_{l}=4 L(l-1) /\left(2 N_{z}^{i}-1\right)-L$ and the Riemann approximation gives:

$$
\begin{aligned}
\frac{1}{2} \int_{-L}^{3 L} \widetilde{T}^{m}(\theta, z, k) \sin \left(\frac{(2 p+1) \pi z}{2 L}\right) \mathrm{d} z & \simeq-\operatorname{Im}\left[\frac{2 L}{2 N_{z}^{i}-1} \sum_{l=1}^{2 N_{z}^{i}-1} i(-1)^{p} \widetilde{T}^{m}\left(\theta, z_{l}, k\right) \exp \left(-i \frac{2 \pi(l-1)}{2 N_{z}^{i}-1}(2 p+1)\right)\right] \\
& =-\frac{2 L}{2 N_{z}^{i}-1} \operatorname{Re}\left[\mathrm{fft}\left[(-1)^{p} \widetilde{T}^{m}\right]_{2 p+1}\right]
\end{aligned}
$$

Thus, by using again the Riemann approximation for the second integral:

$$
B_{n, p}^{k}=\frac{-2}{N_{\theta}^{i}\left(2 N_{z}^{i}-1\right)} \mathrm{fft}\left[\operatorname{Re}\left[\mathrm{fft}\left[(-1)^{p} \widetilde{T}^{m}\right]_{2 p+1}\right]\right]_{n}
$$

Thus, coefficients $A_{n, p}^{k}$ and $B_{n, p}^{k}$ are computed with double fft. The computation time and the accuracy are very dependent on the number of interpolation $N_{\theta}^{i}$ and $N_{z}^{i}$. A compromise should be found. In the paper two options are tested $N_{\theta}^{i}=1000$ and $N_{z}^{i}=100$ for a CPU time of $0.07 \mathrm{~s} /$ cycle and $N_{\theta}^{i}=1000$ and $N_{z}^{i}=1000$ for a CPU time of $0.5 \mathrm{~s} /$ cycle.

\section{References}

[1] Z. Jiang, A. Tieu, Modeling of the rolling processes by a 3-D rigid plastic/ visco-plastic finite element method with shifted ICCG method, Comput Struct 40 (2001) 79-2727.

[2] S. Abdelkhalek, P. Montmitonnet, N. Legrand, P. Buessler, Coupled approach for flatness prediction in cold rolling of thin strip, International Journal of Mechanical Sciences 53 (2011) 661-675.

[3] M. Abbaspour, A. Saboonchi, Work roll thermal expansion control in hot strip mill, Applied Mathematical Modeling 32 (2008) 2652-2669.

[4] X. Zhang, Z. Jiang, A. Tieu, X. Liu, G. Wang, Numerical modelling of the thermal deformation of CVC roll in hot strip rolling, Journal of Materials Processing Technology 130 (2002) 219-223.

[5] G. Zhang, H. Xiao, C. Wang, Three-Dimensional Model for Strip Hot Rolling, Journal of Iron and Steel Research, International 13 (2006) 23-26.

[6] L. Zone-Ching, C. Chang-Cheng, Three-dimensional heat-transfer and thermal-expansion analysis of the work roll during rolling, Journal of Materials Processing Technology 49 (1995) 125-147.

[7] C.-S. Li, H.-L. Yu, G.-Y. Deng, X.-H. Liu, G.-D. Wang, Numerical simulation of temperature field and thermal stress field of work roll during hot strip rolling, Journal of Iron and Steel Research International 14 (2007) 18-21.

[8] P. Montmitonnet, Hot and cold strip rolling processes, Computer methods in applied mechanics and engineering 195 (2006) 6604-6625.

[9] R. Corral, R. Colas, A. Perez, Modeling the thermal and thermoelastic responses of work rolls used for hot rolling steel strip, Journal of Materials Processing Technology 153 (2004) 886-893. 
[10] N. Legrand, D. Weisz-Patrault, N. Labbe, A. Ehrlacher, T. Luks, J. Horsky, Characterization of roll bite heat transfers in hot steel strip rolling and their influence on roll thermal fatigue degradation, Key Engineering Materials (In press).

[11] A. Hacquin, Modelisation thermo-mecanique tridimensionnelle du laminage: couplage bande-cylindres [3D thermomechanical modelling of rolling processes: coupling strip and rolls], Ph.D. thesis, Cemef Ecole des Mines de Paris, 1996. In French.

[12] F. Volle, M. Gradeck, D. Maillet, A. Kouachi, M. Lebouche, Inverse Heat Conduction Applied to the Measurement of Heat Fluxes on a Rotating Cylinder : Comparison Between an Analytical and a Numerical Technique, Journal of Heat Transfer 130 (2008) 1-8.

[13] P. Kotrbacek, M. Raudensky, J. Horsky, M. Pohanka, Experimental study of heat transfer in hot rolling, Revue de Metallurgie-Cahiers d'Informartions Techniques 103 (2006) 333-341. ATS International Steelmaking Conference, Paris, France, dec 15-16, 2005.

[14] W.-L. Chen, Y.-C. Yang, Inverse problem of estimating the heat flux at the roller/workpiece interface during a rolling process, Applied Thermal Engineering 30 (2010) 1247-1254.

[15] F. Volle, D. Maillet, M. Gradeck, M. Lebouche, Semi-analytical inverse heat conduction on a rotating cylinder with Laplace and Fourier transforms, Inverse Problems in Science and Engineering 16 (2008) 655-674.

[16] F. Volle, D. Maillet, M. Gradeck, A. Kouachi, M. Lebouche, Practical application of inverse heat conduction for wall condition estimation on a rotating cylinder, International Journal of Heat and Mass Transfer 52 (2009) $210-221$.

[17] M. Gradeck, A. Kouachi, J. Borean, P. Gardin, M. Lebouche, Heat transfer from a hot moving cylinder impinged by a planar subcooled water jet, International Journal of Heat and Mass Transfer 54 (2011) 5527 -5539 .

[18] D. Weisz-Patrault, A. Ehrlacher, N. Legrand, Evaluation of temperature field and heat flux by inverse analysis during steel strip rolling, International Journal of Heat and Mass Transfer 55 (2012) 629-641.

[19] D. Weisz-Patrault, A. Ehrlacher, N. Legrand, N. Labbe, J. Horsky, T. Luks, Experimental study of interfacial heat flux and surface temperature by inverse analysis with thermocouple (fully embedded) during hot steel strip rolling, Advanced Materials Research 452-453 (2012) 959-963.

[20] N. Legrand, N. Labbe, D. Weisz-Patrault, A. Ehrlacher, J. Horsky, T. Luks, Analysis of roll gap heat transfers in hot steel strip rolling through roll temperature sensors and heat transfer models, Key Engineering Materials 504-506 (2012) 1043-1048.

[21] D. Weisz-Patrault, A. Ehrlacher, N. Legrand, A new sensor for the evaluation of contact stress by inverse analysis during steel strip rolling, Journal of Materials Processing Technology 211 (2011) 1500-1509.

[22] D. Weisz-Patrault, A. Ehrlacher, N. Legrand, Analytical inverse solution for coupled thermoelastic problem for the evaluation of contact stress during steel strip rolling, Applied Mathematical Modelling 37 (2013) 2212-2229.

[23] K. Keanini, Inverse estimation of surface heat flux distributions during high speed rolling using remote thermal measurements, International Journal of Heat and Mass Transfer 41 (1998) 275-285.

[24] T. Loulou, E. Artioukhine, Numerical solution of 3D unsteady nonlinear inverse problem of estimating surface heat flux for cylindrical geometry, Inverse problems in science and engineering 14 (2006) 39-52.

[25] M. Raynaud, J. Bransier, A new finite-difference method for the nonlinear inverse heat conduction problem, Numerical Heat Transfer 9 (1986) 27-42.

[26] P. Hello, J. Vinet, Analytical models of thermal aberrations in massive mirrors heated by high-power laserbeams, Journal de Physique 51 (1990) 1267-1282.

[27] C. Lanczos, Linear differential operators, Van Nostran, Londres and New York, 1961. 
[28] F. Acton, Numerical methods that work, The mathematical Association of America, 1990.

[29] I. Sneddon, Mixed boundary value problems in potential theory, North-Holland Publishing Company, Amsterdam, 1966.

[30] G. Watson, A treatise on the theory of bessel functions, Cambridge University Press, Cambridge, 1944.

[31] I. Gradshteyn, I. Ryzhik, Tables of integrals, Series and Products, Academic Press, New York, 1992. 